Electronic and structural Properties of Metallic Microclusters*

\author{
Amitesh Maiti
}

$I B L-32185$

DE92 016917

Department of Physics, University of California, Berkeley,

and

Materials and Chemical Sciences Division,

Lawrence Berkeley Laboratory,

Berkeley, CA-94720.

April 1992

* This work was supported in part by the Director, Office of Energy Research, Office of Basic Energy Sciences, Materials Sciences Division of the U.S. Department of Energy under contract NO. DE-ACO3-76SF00098. 


\title{
Electronic and Structural Properties of Metallic Microclusters
}

\author{
Amitesh Maiti
}

\begin{abstract}
The first part of this thesis presents a first-order pseudopotential calculation at $T=0$ of the total energy of small sodium clusters of size $N<800$. The calculation is based on a localpseudopotential scheme and local-density correlation and exchange. A temperature-size $(T-N)$ phase-diagram is then derived using the $T=0$ results and Lindemann's criterion for melting. The phase-diagram contains three regions of stability: (1) a liquid (jellium) phase at temperatures above the melting line $T_{M}(N)$ where cluster-stability occurs at electronic magic numbers: (2) a phase related to complete geometrical shells of body-centered-cubic structure at temperatures below the melting line; and (3) a close-packed structure at very low temperatures and sufficiently large $N$. The melting line drops to $T_{M}(N)=0$ for $N<65$, where electronic magic numbers are stable even at $T=0$. The phase diagram reduces asymptotically to the known phases of sodium as $N \rightarrow \infty$, including the known martensitic transformation at $T \sim 5 \mathrm{~K}$. The second and the last part of this thesis consists of a study of small-cluster many-body systems by means of an on-site "local" chemical potential which allows the continuous variation of local electron-density. This method yields a criterion to distinguish particular features of a small cluster that are likely to survive in the large- $N$ thermodynamic limit from those discontinuities that arise only
\end{abstract}


from finite-size effects. The Mott-insulating state, spin-polarized state, and electron-pairing conditions are, in particular, examined. Two foursite Hubbard-model clusters -- a ring and a tetrahedron -- are considered as examples.

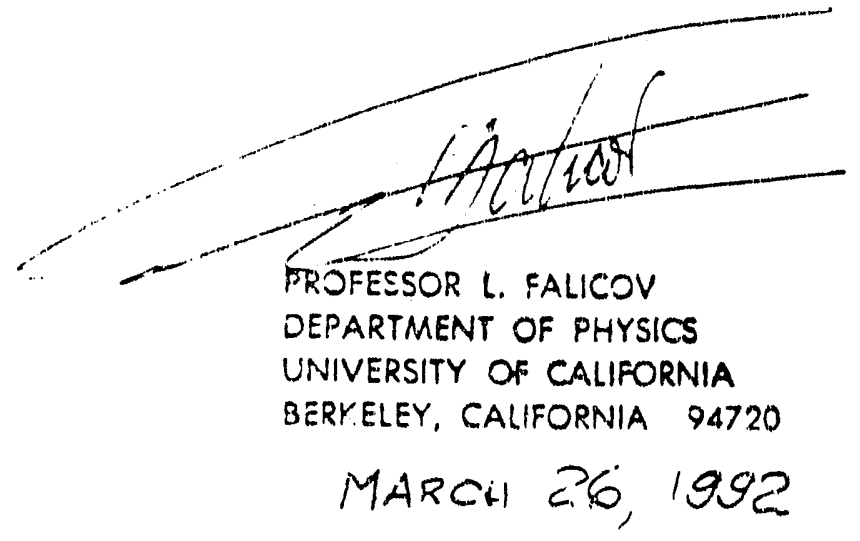




\section{Table of contents}

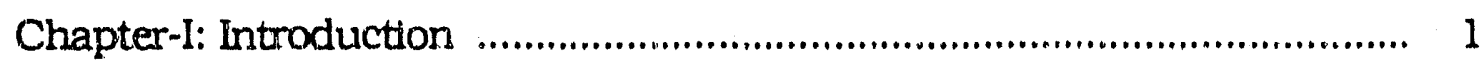
A. Simple metal clusters -.- some experimental background ....................................... 3
B. Theoretical status ....................................................................... 4
C. "Many-body" small clusters ................................................... 7
D. References for chapter- $\mathbb{1}$...................................................... 9

Chapter-II: Pseudopotential calculation of the total energy of small sodium clusters : Cross-over from electronic closed shells to geometrical-structure stability
A. Introduction 12
B. The model 14
C. Calculations and results 20
D. Conclusion 24
E. Appendix to chapter-II 26
F. Raferences for chapter-II 29
G. Tables for chapter-II 32
H. Figures for chapter-II 37

Chapter-III: Phase-diagram for small sodium clusters
A. Introduction 47
B. The model 48
C. Calculation and results 50
D. Conclusion 54
E. References for chapter-III 57 
Chapter-IV: Local chemical potential approach to small-cluster manybody systems
A. Introduction
66
B. Calculations and results 69
C. Discussion 81
D. Appendix-I to chapter-IV 83
E. Appendix-II to chapter-IV 84
F. References for chapter-IV 85
G. Figures for chapter-IV 90 


\section{Acknowledgments}

The intellectually stimulating atmosphere at Berkeley has been an extremely rewarding experience for me. It is impossible to mention, in this short space, all my friends who have made the last four years as pleasant as they have been. To those, not mentioned below, I extend my sincerest gratitude.

First of all I thank my advisor, Prof. Leo Falicov. I really admire his supportive stand from the very beginning, and particularly appreciate his patience and understanding when things were a bit slow during the first year. Working for him has been the most enjoyable both from an academic as well as a human perspective.

I thank the other members of Falicov's group, Darryl Chrzan, Jim Freericks, Andreas Giesekus, Henrik Svensmark, Emilio Artacho, François Willaime, Randy Hood, Antônio da Silva and Sadi Turgut.

I thank all members of the Condensed Matter group I have benefited from long discussions with: Prof. Dan Rokhsar, Ole Christensen, Vitaly Kresin, Michael Surh, Xuejun Zhu and Jing Zhu.

Finally, I thank my family: my mother, whose inspiration and endeavor from my high school days made it all possible; my father and my sister, whose love and affection helped me through rough times; my dear aunt Papai and her family in Arizona, who made me feel like I had never left home; and last but not the least, my wife Pinky, who made the final year of Grad school all the more pleasant with her companionship, understanding and encouragement. 
This thesis is dedicated to my mother, Mrs. Sanchita Maiti. 


\section{Chapter-I : Introduction}

... these atoms exist in the unbound vold, being entirely separate from each other... they differ in shape, size, position, and arrangement ... they move through the void, overtaking each other and colliding ... because they fit together in shape, size, position, or arrangement, they become interlocked and so remain in association...this is the origin of composite bodies ... Leukippos (500 b.c.)

From the ancient times, the human mind has wondered at the unfathomable deptil of the vast universe on one end, and the mysteries of the microworld of "atoms" at the other. Although, it was not possible to think about "internal structure" of atoms even in the middle of last century, it was clear that any macroscopic object was made up of microscopic entities carrying its signature. Only in the first quarter of this century was the internal structure of an isolated atom understood in terms of a negatively charged "cloud" of quantum mechanical particles, the electrons, surrounding a heavy nucleus with a positive charge of equal magnitude. This simple picture set the stepping stone to further studies concerning atoms or aggregates of atoms, and for practical reasons evolved in two extreme directions: (i) Solid State Physics -- the study of solid crystalline objects modeled typically by an infinite periodic array of atoms; and (ii) Atomic and Molecular Physics (and Chemistry) -... the study of a single atom or a molecular cluster. What made these two branches of study almost completely separate disciplines of physics is the large differences in the physical nature of electrons in the two systems. As an illustration let us consider the element iron. An isolated iron atom has all its electrons confiner to 
well within a few atomic units, and the net electron spin in the ground state is $4.0 \mu_{B}$. In an ideal metallic solid (free of surface, domain boundaries or point defects, and at $T=0$ ), on the other hand, all the valence (4-s and 3-d) electrons are believed to be completely itinerant throughout the entire solid, and the average electron spin per atom is only $2.2 \mu_{B}$. Development of theoretical and experimental techniques in the study of surfaces, defects, interfaces and low-dimensional systerns in the last couple of decades have raised many interesting questions and problems that directly fall in neither of the two categories of physical studies mentioned above, but rather somewhere in between. The physics of clusters, elemental or compound, of size from a few atoms to a few thousand, is primarily aimed at addressing questions regarding how the physical nature of electrons and the accompanying structure of solids evolve from an isolated atomic or molecular limit to the bulk as one goes up in cluster size by successive addition of atoms. Many interesting questions come to mind : Are the electrons in a silicon cluster itinerant below a critical size? What is the critical size of a given cluster above which the arrangement of atoms begin to look like a bulk structure? Is it possible to have a martensitic transformation or a metal-insulator transition as a function of clustersize? Or, in case of a magnetic cluster, how does the magnetization per atom change as one goes to larger and larger clusters? In order to understand the development of different kinds of crystals, clusters with inherently different electronic structures are being investigated. A broad classification of the inorganic clusters, along with the commonly accepted intuitive picture of them are listed below:

(i) "Metallic" clusters (simple alkali metal clusters -.- described by 
free electrons, jellium model);

(ii) "Inert gas" clusters ( -.- billiard ball atoms, pairwise Van der Waals interaction, icosahedral packing);

(iii) "Covalent clusters" (e.g. C lthe $\mathbf{C}_{60}$ clusters or buckeyballs being of particular interest at the time of this writing), $\mathrm{Si}, \mathrm{Ge}$ clusters -.- ball and stick models);

(iv) "Noble-metal clusters" ( -.. metallic, with a discernible influence of the $d$-electrons in the form of $s-d$ mixing);

(v) "Transition-metal clusters" (e.g. Fe, Co clusters --- magnetic, $d$ electrons play a major role).

A major part of this thesis (chapters II and III) deals with electronic and structural properties of the simple metal clusters of class (1) listed above. It is therefore relevant to review some of the experimental and theoretical results on these clusters obtained over the last few years.

\section{A. Simple metal clusters --- some experimental background}

The recent interest of the scientific community in metallic clusters started in 1984 from a major discovery 1,2 of Walter Knight and his collaborators at the University of California. Berkeley. Using a supersonic nozzle source the group produced stable sodium clusters by seeding an inert carrier gas (Helium) with a low concentration of nearly saturated metal vapor, which was then cooled by adiabatic expansion of the gas in vacuum. Clusters were formed when cooling resulted in supersaturation. The mass of the clusters could then be determined using a time-of-flight mass spectrometer. The relative 
number of clusters of different sizes could be easily determined by adjusting the spectrometer to various mass-selective "windows". The "abundance spectrum" thus obtained by Knight's group for sodium clusters up to a size $N \sim 100$ had sharp local maxima --- clusters of certain particular sizes were found to be strikingly more abundant than the others. Since the relative abundance of a given cluster size is related directly to its stability, the spikes in Knight's data represented the most stable clusters under experimental conditions. Usually, such stable structures are thought of as those for which the atoms, considered stationary, form a particularly robust low energy configuration. However, the amazing finding of Knight's experiment was that the series of stable. cluster-sizes $(N=8,20,40,58,92, \ldots)$ corresponded to filled electronic angular-momentum shells in a spherically symmetric geometry, and did not seem to depend on the details of ionic positions. The resulting picture was very similar to what had been known about nuclear stability for a long time : the nucleus is relatively more stable for certain numbers of nucleons, called the "magic numbers", corresponding to filled "nuclear shells". In analogy, the term "electronic magic numbers" was coined to describe the most stable cluster-sizes in Knight's experiment. More recently, in 1990, a group in Copenhagen headed by S. Bjфrnholm ${ }^{3}$. and a group in Stuttgart headed by T. P. Martin 4,5 have extended the Berkeley experiment to larger clusters and clusters of other alkali metals. While the Danish group observed electronic magic numbers for clusters up to a size of several hundred, the German group made another startling discovery : while the small sodium clusters (up to $N$ $\sim 1,500$ ) show peaks in the abundance spectrum at electronic magic 
numbers, the most stable clusters for $N$ between about 1,500 and their upper limit of observation $(22,000)$ are those governed by geometrical closing of icosahedral structures.

\section{B. Theoretical status}

In order to understand theoretically cluster stability at electronic magic numbers, most calculations so far have embarked upon the philosophy that detailed ionic positions are not important for low-energy structures. This starting assumption, coupled with the fact that electron angular-momentum shells play a prominent role, led theorists to approximate the ions not by discrete, charged particles, but by a sphere of evenly smeared out, positively charged "jelly". Such a conceptual object, popularly known as "jellium", was meant to provide a spherically symmetric environment to the itinerant electrons. With the jellium constructed, density functional theory was used to minimize the total energy of the cluster and determine selfconsistently the associated electronic charge-density. Such a calculation, known as the spherical jellium background model6-8 (SJBM) could generate stability at the electronic magic numbers. However, it suffers from some serious drawbacks -.-

(1) the ions are discrete charges, and cannot be replaced by a positive background, particularly in a "solidlike" cluster, where the ions vibrate with a small amplitude about their mean positions; and (2) the spherical jellium model, by its very construction, cannot explain stability at "geometrical" shell closings as is observed in Martin's experiments.

It is clear that in order to understand a cross-over of cluster 
stability from electronic magic numbers to those governed by geometrical shell closings of atoms, one rnust include, in a total energy calculation, discrete tonic cores from the very beginning. Moleculardynamics or similar calculations ${ }^{914}$ are able to treat the problem of determining the lowest energy configuration of the ions. However, such calculations are computationally taxing, and even with present day computers one cannot go to clusters of more than $\sim 50$ atoms. In order to address the kind of cross-over described above, it is necessary to adrpt some kind of an approximate scheme ${ }^{15}$.

The main points of the approach taken in this thesis, and described in technical detail in chapter-II, are:

(1) The electronic charge-density is calculated in an effective potential due to a positive jellium background.

(2) The distortion in the calculated electronic charge-density due to actual discreteness of lons and the accompanying change in energy are taken into account in the first-order perturbation of a local pseudopotential.

(3) Density functional theory is used to calculate the total energy; electron correlation and exchange are treated in the local density approximation.

(4) The electron-background interaction of a jellium model calculation is now replaced by the bare Coulomb interaction of electrons with discrete lons, while the term corresponding to background interaction with itself in an SJBM is relegated to calculating the ion-ion Madelung energy.

(5) The ions are displaced in a fixed electromic background in search for a configuration of minimum total energy. 
Chapter-II describes a zero-temperature total energy calculation on sodium clusters of size up to $\sim 200$. A cross-over from stable electronic magic numbers to stable numbers corresponding to geometrically closed structures occurs at $N \sim 100$. Chapter III extends the calculation to finite temperatures and larger sizes (up to $N \sim 800$ ). It is found that the zero-temperature structure for most stable clusters of size $N>100$ is always a "solidlike" geometrically closed, closepacked one. The experimentally observed electronic magic numbers, on the other hand, are "liquidlike" clusters. This structure is caused by the relatively rapid ionic motion at the experimental temperatures; treating them in a jellium background model is not as drastic an approximation as seems at first thought.

\section{C. "Many-body" small clusters}

Apart from "real" atomic clusters described above. the concept of "hypothetical" small clusters has also been extremely useful in a completely different context. Ever since the inception of Quantum mechanics, a major part of Solid-State (Condensed-Matter) and Statistical Physics have been involved in solving Model Hamiltonians on lattices of different structures and dimensions. Simple models describing complicated many-body effects in a large range of materials have been particularly pupular over the last thirty years. Some of these, for instance, are the ones associated with names of Ising. Heisenberg, Hubbard, Anderson, Falicov-Kimball, Kondo and so on. Unfortunately, even these simplified models are not amenable to exact solutions except under very special circumstances which are usually not very meaningful. Traditional ways out have involved taking recourse to 
direct ${ }^{16-18}$ or diagrammatic perturbation methods $\mathbf{1 9 , 2 0}$ or making a one-particle approximation in some mean-field scheme 21 and solving the problem approximately on a full, infinite lattice. Such a procedure, however, often leads to wrong results in systems where electron correlation is important. An alternative approach has been to keep the electron-correlation completely in the Hamiltonian, and solve its spectrum exactly on a small "cluster" that is a representative subset of the lattice one originally starts with $\mathbf{2 2 , 2 3}$. The mail drawback of this approach is that size effects are overwhelming in such a system, even with periodic boundary conditions, and one might obtain results that probably cannot be generalized to the large- $N$, thermodynamic limit. This point is taken up in chapter- $\mathrm{N}$ where the Hubbard model is exactly solved on a couple of small clusters. Thermodynamic localoccupation averages and local-number-occupation fluctuations are studied by introducing a single-site chemical potential at a particular site $^{24}$. This procedure allows the study of differential properties of thermodynamic functions by providing continuous variation of local occupation. The method gives a criterion to distinguish particular features of the small cluster that are likely to survive in the thermodynamic limit from those discontinuities that are characteristic of the finiteness of the cluster and the resulting discreteness of the energy-spectrum. In particular the Mott-Insulator state (a discontinuity of chemical potential at a particular electron-density) can be clearly tested this way. Similar indications are obtained for spin-polarized states and for particle-pairing conditions. 


\section{References for chapter-I}

1. W. D. Knight, K. Clemenger, W. A. de Heer, W. A. Saunders, M. Y. Chou, and M. L. Cohen, Phys. Rev. Lett. 52. 2141 (1984).

2. For a detailed review and other references for earlier work, see W. A. de Heer, W. D. Knight, M. Y. Chou, and M. L. Cohen, in Soltd State Physics, edited by H. Ehrenreich, F. Seitz, and D. Turnbull (Academic, New York, 1987), vol. 40, pp. 93-181.

3. S. Bjфrnholm, J. Borggreen, O. Echt, K. Hansen, J. Pedersen, and H. D. Rasmussen, Phys. Rev. Lett. 65, 1627 (1990); Z. Phys. D 19. 47 (1991).

4. H. Göhlich, T. Lange, T. Bergmann, and T. P. Martin, Phys. Rev. Lett. 65, 748 (1990); Mod. Phys. Lett. 5, 101 (1991); Z. Phys. D 19. 117 (1991).

5. T. P. Martin, T. Bergmann, H. Göhlich, and T. Lange, Chem. Phys. Lett. 172, 209 (1990), Z. Phys. D 19, 25 (1991).

6. M. L. Cohen, M. Y. Chou, W. D. Knight, and W. A. de Heer, J. Phys. Chem. Solids 91, 3141 (1987).

7. W. Ekardt, Phys. Rev. B 29, 1558 (1984).

8. T. Lange, H. Göhlich, T. Bergmann, and T. P. Martin, Z. Phys. D 
19. 113 (1991).

9. R. Car, and M. Parrinello, Phys. Rev. Lett. 55, 2471 (1985).

10. P. Ballone, W. Andreoni, R. Car, and M. Parrinello, Europhys. Lett. 8, 73 (1989).

11. R. Kawai, J. H. Weare, Phys. Rev. Lett. 65, 80 (1990).

12. O. Sugino, and H. Kamimura, Phys. Rev. Lett. 65, 2696 (1990).

13. O. B. Christensen, K. W. Jacobsen, J. K. Nфrskov, and M. Manninen, Phys. Rev. Lett. 66, 2219 (1991).

14. J. Y. Yi, J. O. Dirk, and J. Bernholc, Phys. Rev. Lett. 67, 1594 (1991).

15. A. Maiti, and L. M. Falicov, Phys. Rev. A 44, 4442 (1991).

16. P. W. Anderson, in Solid State Physics, edited by F. Seitz, and D. Turnbull (Academic, New York, 1963), Vol. 14, p. 99.

17. J. R. Schrieffer, and P. A. Wolff, Phys. Rev. 149, 491 (1966).

18. A. B. Harris, and R. V. Lange, Phys. Rev. 157, 295 (1967).

19. V. M. Galitskii, Sov. Phys. JETP 7, 104 (1958). 
20. D. Bohm, and D. Pines, Phys. Rev. 92, 609 (1953); M. Gell-Mann and K. Brueckner, Phys. Rev. 106, 364 (1957).

21. P. Coleman, Phys. Rev. B29, 3035 (1984).

22. L. M. Falicov, in Recent Progress in Many-body Physics, edited by A. J. Kallio, E. Pajanne, and R. F. Bishop (Plenum, New York, 1988), Vol. 1; J. Callaway, Physica B 149, 17 (1988).

23. J. K. Freericks, Ph.D. Thesis, University of California, Berkeley, May 1991, and references therein.

24. A. Maiti, and L. M. Falicov, Phys. Rev. B 43, 788 (1991). 


\section{Chapter-II : Pseudopotential calculation of the total energy of small sodium clusters: Cross-over from electronic closed shells to geometrical-structure stability}

\section{A. Introduction}

Ever since the discovery by Knight et al. ${ }^{1}$ of quantal shell structure in small droplets of sodium metal, with characteristic "magic numbers", metal-cluster physics has become an active field of theoretical and experimental investigation. Many properties of a large variety of clusters have been examined ${ }^{2}$.. mass abundance spectra, fragmentation spectra and binding energy, supershell structure ${ }^{3}$, iorization potential, photoelectron spectra and electron affinity, static electric polarizability 4 , plasma resonance spectra, and thermal properties $^{5}$, to name a few. Because a cluster lies somewhere between a solid and a molecule, the problem has been approached from two extreme directions -- solid-state theorists employ modified quantum models of bulk solid $\mathbf{2 , 6 , 7}$, whereas molecular chemists and atomic physicists attempt molecular-dynamical calculations in which one builds up clusters atom by atom. A compelling stimulus throughout has been the desire to understand how an extended crystalline solid develops from growing cluster aggregates. Two major experimental results in this regard have been (i) the observation of cluster stability exactly at the so-called electronic magic numbers $1.8-11$.- which correspond to electronic shell-closings -- for cluster sizes less than 200 , and (ii) the more recent discovery by Martin et al.12 of clusterstability at magic numbers corresponding to closed-packed atomic shell arrangements in icosahedral or cuboctakedral packings for 
clusters of size between $\sim 1,500$ and 22,000 . The stability characteristics of the small clusters are well supported by the calculations of Cohen et al.13 and of Ekardt ${ }^{14}$, based on the selfconsistent jellium background model (SJBM). Although jellium-like mean-field calculations exist for cluster-sizes of the order of several hundred ${ }^{15}$, in order to study properly this transition in stability from electronic to atomic magic numbers, one needs to incorporate the atomic structure, i.e. the ions must be explicitly included for any cluster-size. One would, in principle, like to approach the problem by self-consistently determining atomic configurations and the associated electronic charge density that yield the minimum total energy for each cluster size in question. Several systematic methods have been developed in this regard by solid-state theorists 6 and molecular chemists ${ }^{16}$. Unfortunately, because of the enormous computational tax, these calculations as of now are limited to sizes well below a hundred ions. Work has also been done on statistical descriptions of the electronic level structure ${ }^{17}$ and the asymptotic size-dependence of the energy in large clusters 18 . However, no successful theory of the observed transition from electronic to atomic dominance in the structure have appeared in print, as far as the author is aware.

The present chapter attempts to study these clusters by means of a pseudopotential approach, for which one can easily tackle clusters of size up to a few thousand with reasonable computational effort. By comparing the numerical results for total energy per atom obtained at several electronic and atomic magic numbers, a pattern emerges which clearly shows that for small clusters the electronic structure dominates, whereas at larger sizes the closing of ionic crystal-like 
shells becomes paramount. A cross-over is thus found. It should be pointed out that in all cases the clusters are small, in the traditional sense of the concept, since all atoms are, in all cases, within a few Angstrom from the surface, i.e. far away from what can be considered the bulk limit.

\section{B. The model}

The model consists of approximating the self-consistent KohnSham (KS) potential observed by each electron with an infinite well, the shape of whose boundary is defined, in principle, by the surface of the outermost ions in the cluster. Because of the observation that the spherical jellium model works well for small clusters $(N<200)$, coupled to the convenience of separability of co-ordinates in a spherical geometry, the electron confining regions for any cluster size are taken to be infinite spherical wells. Two different schemes of choosing the cluster sizes for the purpose of calculation are considered, in conformity with the spherical boundary :

(A) The clusters representative of the geornetrically closed shells of atoms (hereafter described as the ones with atomic magic numbers) are made up of finite, perfect periodic lattices cut off by the surface of the spherical well and then allowed to relax; and

(B) The clusters representative of electronic magic numbers consist of the ions placed within the electron confining spherical well so as to minimize (numerically) the total energy of the cluster. 
For the atomic magic number structures four different types of finite lattices have been considered in this calculation :

(A1) Finite body-centered-cubic (bcc) lattices (the bulk structure of sodium at low temperatures) with an atom at the center of the electron cos:fining sphere;

(A2) Finite face-centered-cubic $(f c c)$ lattices with the sphere centered at an atom in the lattice (henceforth referred to as Type-I $f c c$ clusters);

(A3) Finite fcc lattices with the sphere centered at the center of a conventional unit cube (referred to hereafter as Type-II fcc clusters) and,

(A4) Finite $f c c$ lattices with the sphere centered at the center of a tetrahedron of nearest-neighbor atoms (Type-III fcc clusters).

Each type of cluster, initially arranged according to schemes (A1-4) and (B) described above, correspond to different distributions of atoms in the sphere and, accordingly, yield separate sets of magic numbers. There are a few numbers that are common to two or more cluster types, e.g. 68, which is common to schemes (A3), (A4) and $(B)$, and 92, common to schemes (A4) and (B).

All schemes (A1-4) and (B) are starting points for relaxing the structures in search of an energy minimum. The relaxation is such that certain constraints, listed below, are obeyed.

(1) No two ions lie within a distance $2 r_{c}$ of one another, $r_{c}$ being the effective 'hard-core' radius for the metallic ion under consideration, 
i.e. sodium. One could estimate $r_{c}$ from the effective two-body interaction between two sodium atoms in a lattice obtained by secondorder perturbation theory of the pseudopotential ${ }^{19}$. The curve resulting from such a calculation 20 is shown in Fig. 2.1. In this way, $2 r_{c}$ has been estimated to be $\sim 0.82 a$, where $a=4.225 \dot{A}$ is the bulk latticeconstant for sodium in the bcc structure.

(2) No ions should lie outside the effective jellium surface, a sphere of radius $R_{+}$.

(3) The jellium (uniform positive-charge background) sphere, of radius $R_{+}$lies inside an infinite-potential spherical well that confines the electrons; the latter has radius $R$ (Fig. 2.2). The difference in radii $\delta=$ $R-R_{+}$physically represents the 'decay length' of electronic wavefunctions outside the actual surface of a solid or cluster, which is bound by a finite potential well. The value of $(\delta / a)$ is taken to be a constant, equal to 0.29 , independent of cluster size. This is a very good approximation 21 , as seen from the SJBM results of Ref. 14.

Lattice relaxations that obey the above constraints are allowed on the way to finding the lowest energy states of the various clusters.

Several comments are relevant :

(1) The electronic charge-density calculated here is not far from that calculated by SJBM. A direct comparison of the graphs in Fig. 2.3 with counterparts of those in Figure 3 of Ref.14 makes this fact apparent.

(2) The modification of the electronic wave-function at the ionic sites 
is taken into account in the first-order perturbation of the pseudopotential. Any higher-order perturbation, which is computationally forbidding, should have (for alkali-atom clusters) an insignificant effect on the trend of results for the total energy.

The total energy $E_{t o t}$ per atom, in the model considered here, is a sum of several contributions given by:

$$
E_{t o t}=E_{e l-e l}+E_{e l-t o n}+E_{i o n-t o n}+E_{k i n}+E_{x c}
$$

The various terms on the right hand side of the above equation are self-explanatory and are calculated for a cluster of size $N$ using the following formulas :

For the electron-electron Coulomb interaction

$$
E_{e l-e l}=N \frac{e^{2}}{2} \int \frac{\rho(\boldsymbol{r}) \rho\left(\boldsymbol{r}^{\prime}\right)}{\left|\boldsymbol{r}-\boldsymbol{r}^{\prime}\right|} d \boldsymbol{r} d \boldsymbol{r}^{\prime} \quad
$$

for the electron-ion Coulomb interaction

$$
E_{e l-i o n}=-e^{2} \sum_{i} \int \frac{\rho(\boldsymbol{r})}{\left|\boldsymbol{r} \cdot \boldsymbol{R}_{i}\right|} d \boldsymbol{r}+E_{p s}(1) .
$$

where $E_{p s}{ }^{(1)}$ is defined below; the ion-ion Coulomb interaction

$$
E_{\text {ton-ion }}=\frac{e^{2}}{2 N} \sum_{i \neq j} \frac{1}{\left|\boldsymbol{R}_{t}-\boldsymbol{R}_{j}\right|} ;
$$

for the electron kinetic energy 


$$
E_{k i n}=-\frac{\hbar^{2}}{2 m N} \sum_{\alpha, \sigma} \int_{\psi_{\alpha \sigma}^{*}(\boldsymbol{r}) \nabla^{2} \psi_{\alpha \sigma}(\boldsymbol{r}) d \boldsymbol{r} ;}
$$

and for the electron exchange and correlation energy in the localdensity approximation

$$
E_{x c}=\int d \boldsymbol{r} \rho(\boldsymbol{r}) \varepsilon_{x c}(\rho(\boldsymbol{r}))
$$

In equations $(2.2-6) \psi_{\alpha \sigma}(r)$ stands for the wave-function of the electron in the quantum state $\alpha$ with spin $\sigma$. Also introduced above are the electronic number density $\rho(\boldsymbol{r})$ normalized to one,

$$
\begin{aligned}
& \rho(\boldsymbol{r})=\frac{1}{N} \sum_{\alpha, \sigma}\left|\psi_{\alpha \sigma}(\boldsymbol{r})\right|^{2}, \\
& 1=\int d \boldsymbol{r} \rho(\boldsymbol{r})
\end{aligned}
$$

the first-order pseudopotential contribution $E_{p s}{ }^{(1)}$ is

$$
E_{p s}(1)=\sum_{t} \int_{d r} \rho(\boldsymbol{r}) V\left(\left|r-R_{t}\right|\right),
$$

with the local pseudopotential function $V(r)$ given by 22

$$
\begin{aligned}
& V(r)=\frac{e^{2}}{r_{0}} u, \quad r<r_{0} . \\
& -\frac{e^{2}}{r}, \quad r>r_{0} \text {, }
\end{aligned}
$$


where $u$ and ro are phenomenological parameters; and the exchangecorrelation local density functional is

$$
\varepsilon_{x c}(\rho(\boldsymbol{r}))=-0.9162 / r_{s}(\boldsymbol{r})-0.0666 G\left[r_{s}(\boldsymbol{r}) / 11.4\right],
$$

where the quantity $r_{s}(r)$ and the function $G$ are given by 14 :

$$
r_{s}(\boldsymbol{r})=\frac{1}{a_{B}}[3 / 4 \pi \rho(\boldsymbol{r})]^{1 / 3}
$$

and where $a_{B}=0.529 \AA$ is the Bohr radius, and

$$
G(x)=\left[\left(1+x^{3}\right) \ln \left\{1+\frac{1}{x}\right\}-x^{2}+\frac{x}{2}-\frac{1}{3}\right] .
$$

The values of the pseudopotential parameters $u$ and $r_{0}$ are taken from the results known 22 in the thermodynamic limit:

$$
u=-0.3632 R y, \text { and } r_{0}=1.097 \AA
$$

respectively. The only difference between the small clusters and the bulk arises from the drastic changes in the electronic chargedensities. 


\section{Calculations and results}

For clusters corresponding to electronic magic numbers there is complete spherical symmetry in the charge density of the electrons i.e. $\rho(\boldsymbol{r})$ is only a function of $r=|\boldsymbol{r}|$. This simplifies the computation enormously, because the integrals in (2.2) and (2.3) now get reduced respectively to one and two-dimensional integrals. A further considerable reduction in computational effort occurs in the calculation of $E_{e}$ l-ton when one relaxes the ion positions (in any scheme) against the stationary spherically symmetric electronic background to get to the lowest energy configuration. Unfortunately, the charge density arising from electrons at the outermost -- partially filled -.- shell (i.e. the Fermi level) does not possess a complete spherical symmetry for clusters with numbers other than the electronic magic numbers. However, simple electrostatic considerations show that, for large $N$, the correction to the total energy per atom. if one symmetrically averages over all electron states at the Fermi level in configurations with partially filled outer electronic shell, can be easily estimated. It is of the order of $\Delta E_{e l-e l} \sim$ $0.1\left(n / N^{2}\right) E_{e l-e l,}$ as seen in the Appendix to this chapter; here $n$ is the smallest of the numbers of filled and unfilled states in the shell at the Fermi level. In most situations $n \ll N$, and spherical averaging over all states at the Fermi level involves errors which are always tolerable and usually very small. The calculations were, therefore, performed with spherically averaged charge distributions. Tables 2.1-5 respectively display resul's for the various components of energy and the total energy per atom for a set of magic numbers corresponding to filled electronic and atomic shells for the different cluster types considered. 
Fig. 2.4 graphically displays the results for the total energy per atom as a function of $N$ for both electronic and atomic magic numbers. The convex hulls of the lowest energies found for the A(electronic) and B(atomic) magic numbers are drawn in Fig. 2.5. A global "hull" encompassing all calculations, has a well defined transtition from the "A" hull to the "B" hull at $N \sim 100$. These hulls, similarly to the ones drawn in the theory of heterogeneous alloys, corresponds to states of total stability under the assumption of conservation of the number of clusters, i.e. under the assumption that clusters can only change in relations of the type

$$
c\left(N_{1}\right)+c\left(N_{2}\right) \rightarrow c\left(N_{3}\right)+c\left(N_{4}\right) .
$$

where,

$$
N_{1}+N_{2}=N_{3}+N_{4} \text {. }
$$

and not of the type of fusion or fission reactions, such as

$$
\begin{aligned}
c\left(N_{1}\right)+c\left(N_{2}\right) & \rightarrow c\left(N_{3}\right), \\
N_{1}+N_{2} & =N_{3},
\end{aligned}
$$

or

$$
\begin{gathered}
c\left(N_{1}\right) \rightarrow c\left(N_{2}\right)+c\left(N_{3}\right), \\
N_{1}=N_{2}+N_{3} .
\end{gathered}
$$

The clusters on the global hull are, in any case, very stable clusters over an extencied local size.

There is a clear cross-over of cluster stability from electronic magic numbers at smaller cluster-sizes to the atomic magic numbers at larger cluster-sizes. The cross-over appears to occur at $\mathrm{N} \sim 100$. A number of key features from the numerical results presented in tables 2.1-5 are to be noted: 
(1) For very small clusters $(N<50)$ the kinetic energy $E_{k i n}$ plays a very important role in favoring stability at electronic rather than atomic magic numbers in their vicinity. Closed e:actronic shells always yield lower kinetic energy per atom than open ones.

(2) A complete outer atomic shell structure always lowers the electrostatic part of the energy. For this reason, even for cluster-sizes $N<100$-- dominated by the electronic magic numbers -- clusters with atomic magic numbers close to one with an electronic magic number have large binding energies. The most unstable clusters are the ones with near half-filled $d, f$ or $g$ shells of electrons. This clearly shows that even though atomic positions are extremely important for any cluster-size, the stability for small cluster size is completely dominated by the closing of the electronic shells.

(3) For sizes $N>100$ the most stable clusters are clearly not those corresponding to the electronic magic numbers. Although it is hard to detect any underlying pattern of atomic shell closings in this region, the most stable clusters are always found to belong to one of the three fcc structures built according to schemes (A2-4). Investigation on clusters of larger sizes seems to indicate that the ground state of a "solidlike" cluster of any size is always a close-packed one.

(4) For $N<100$, the binding energy per atom for the electronic magic numbers increases, in general, with increasing cluter-size. For the $N>100$ region the situation is more complicated as supershell structures might come into play 3,15 . 
(5) In the region where electronic magic numbers dominate $(N<100)$ the lattice always relaxes inward, so that the effective inter-atomic distances are smaller than the corresponding value for the bulk. This fact is in agreement with the experimentally observed lattice shrinkage 23 for small clusters. As the cluster size increases, the inward lattice relaxation becomes gradually smaller. Thus the equilibrium lattice constant increases from about $96 \%$ of the bulk value for the smallest clusters to about $99 \%$ for cluster-sizes $~ 200$.

(6) In contrast to the results of the spherical jellium calculations, where the total energy rapidly converges to the bulk value, the present calculations clearly show that even for sizes up to -200 the total electrostatic interaction energy is very different from the bulk value. The reason is that all the atoms, even in the largest clusters considered here, are only a few Angstroms from the surface. This surface dominance is also reflected in the sizeable non-uniformity in the electronic charge-density shown in Figure 1. 


\section{Conclusion}

A total energy calculation based on the local pseudopotential scheme, with correlation and exchange energies taken in the local density functional approach, yields a clear transition in the stability regime of small sodium clusters. For small clusters, $N<100$, clusters corresponding to closed electronic shells (electronic magic numbers) are considerably more stable than all others in their vicinity. For larger numbers, $N>100$, the most stable clusters are those for which the geometric configuration of the ions correspond to well defined polyhedral configurations (atomic magic numbers). A major effect contributing to this transition is the dominance of electronic kinetic energy terms for low $N$, and the electrostatic contribution at large $N$. The stability of the highly coordinated $f c c$ over $b c c$ clusters indicates a close-packed ground state for "solidlike" clusters of all sizes up to the bulk limit. The main reason for obtaining the transition of stability from electronic to atomic magic numbers at a size much smaller than that observed experimentally is neglecting the effect of finite temperature; experiments are done not at $T=0$ for which the present calculation is applicable, but on rather "hot" clusters, pretty much at and above the "melting" temperatures of clusters. Finite temperature effects are taken up in detail in Chapter III of this thesis. The zerotemperature calculations described here could possibly be made more accurate by using a more realistic Kohn-Sham potential e.g. a finite square-well or the Woods-Saxon potential ${ }^{3}$ as used in nuclear physics. Use of a simple infinite well approximation is resporsible for the electronic charge density differ in finer details, despite agreeing in overall features with the SJBM results ${ }^{14}$. 
It should be mentioned that the cross-over effect is a consequence of a smooth relative change in the influence of various contributions, and therefore the transition cannot be considered to be sharp. It is possible that "beats" between the two effects could be observed with an electronic magic number becoming observable between (and probably sufficiently removed from) consecutive atomic magic numbers. It must be finally remarked that the calculation has not attempted to determine the overall shape of the cluster of absolute minimum energy : it has simply demonstrated that geometrical structural considerations overtake electronic-shell arguments when the number of atoms in the cluster becomes larger than a typical "critical" value. 


\section{E. Appendix to chapter-II.}

This appendix gives an estimate of the error involved in the total energy calculation when one uses a spherically symmetrized charge density of electrons, by averaging over the states at the last (partially occupied) level. The two contributions to the total energy per atom that are most affected are $E_{e l-e l}$ and the electrostatic part of $E_{e l-i o n}$. The pseudopotential contribution $E_{p s}{ }^{(1)}$ and the exchangecorrelation energy $E_{x c}$ are themselves at least an order of magnitude smaller than either of the two electrostatic terms; corrections to these terms are negligibly small.

Let $\delta \rho(\boldsymbol{r})$ be the deviation at position $r$ of the actual total number density of the electrons per ion from the used spherically symmetric one. Clearly.

$$
\int \delta \rho(\boldsymbol{r}) d \boldsymbol{r}=0 .
$$

Now, it is evident that the contribution to the total number density arising from all closed shells of electrons, i.e. due to those states below the Fermi level, is already spherically symmetric. Therefore $\delta \rho(\boldsymbol{r})$ arises only from those electrons on the outermost shell. Therefore $\delta \rho(\boldsymbol{r})$ can be written as the difference of the number density of the electrons at the Fermi level from the spherically symmetric electronic number density obtained by averaging over all states at the Fermi level. The same quantity could be equivalently written in terms of unoccupied states at the Fermi level. It is convenient to use the description in terms of either occupied or ampty states, whichever number is smaller at the Fermi level. Let this number be $n$. 
It should be noted that separability in the radial co-ordinate $r$ and angular variables $\Omega \equiv(\theta, \varphi)$ allows a decomposition of $\delta \rho(r)$ into two factors:

$$
\delta \rho(\boldsymbol{r})=f(r) g(\Omega),
$$

where $f(r)=\left|j_{v}\left(k_{v l} r\right)\right|^{2}$ is the same for all electrons at the Fermi level characterized by the principal and total angular-momentum quantum numbers $n$ and $l$ respectively; here $j_{v}$ is the spherical Bessel's function of order $v$ and $k_{v l}$ is the $(l+1)^{\text {th }}$ zero of $j_{v}$.

Equations (A.1) and (A.2) therefore imply,

$$
\int g(\Omega) d \Omega=0 .
$$

The correction to the electron-electron contribution Fel-el is given by,

$$
\begin{gathered}
\Delta E_{e l-e l}=N \frac{e^{2}}{2} \int \frac{\left\{\rho_{O}(r)+\delta \rho(\boldsymbol{r})\right\}\left\{\rho_{O}\left(\boldsymbol{r}^{\prime}\right)+\delta \rho\left(\boldsymbol{r}^{\prime}\right)\right\}}{\left|\boldsymbol{r}-\boldsymbol{r}^{\prime}\right|} d \boldsymbol{r} d \boldsymbol{r}^{\prime} \\
-N \frac{e^{2}}{2} \int \frac{\rho O(r) \rho O\left(\boldsymbol{r}^{\prime}\right)}{\left|\boldsymbol{r}-\boldsymbol{r}^{\prime}\right|} d \boldsymbol{r} d \boldsymbol{r}^{\prime}, \\
=N e^{2} \int \frac{\rho_{O}\left(\boldsymbol{r}^{\prime}\right) \delta \rho(\boldsymbol{r})}{\left|\boldsymbol{r}-\boldsymbol{r}^{\prime}\right|} d \boldsymbol{r} d \boldsymbol{r}^{\prime}+N \frac{e^{2}}{2} \int \frac{\delta \rho(\boldsymbol{r}) \delta \rho\left(\boldsymbol{r}^{\prime}\right)}{\left|\boldsymbol{r}-\boldsymbol{r}^{\prime}\right|} d \boldsymbol{r} d \boldsymbol{r}^{\prime} .
\end{gathered}
$$

where $\rho_{O}(r)$ is the spherically symmetrized number density of electrons per ion obtained by averaging over all states at the Fermi level.

Since 


$$
V(r)=\int \frac{\rho_{0}\left(r^{\prime}\right) d r^{\prime}}{\left|r-r^{\prime}\right|}
$$

is a function of $|\mathbf{r}|=r$ only, the first term on the right hand side of the last equation becomes,

$$
N e^{2} \int \delta \rho(\boldsymbol{r}) V(r) d \boldsymbol{r}=N e^{2} \int f(r) V(r) r^{2} d r . \int g(\Omega) d \Omega=0
$$

where equation (A.3) has been used. Therefore.

$$
\Delta E_{e l-e l}=N \frac{e^{2}}{2} \int \frac{\delta \rho(\boldsymbol{r}) \delta \rho\left(\boldsymbol{r}^{\prime}\right)}{\left|\boldsymbol{r}-\boldsymbol{r}^{\prime}\right|} d \boldsymbol{r} d \boldsymbol{r}^{\prime}>0
$$

Also,

$$
\Delta E_{e l-i o n}=e^{2} \sum_{t} \int \frac{\delta \rho(\boldsymbol{r})}{\left|\boldsymbol{r} \cdot \boldsymbol{R}_{i}\right|} d \boldsymbol{r}
$$

where corrections to $E_{p s}(1)$ have been neglected.

It is important to note that $\Delta E_{e l-e l}$ is always positive

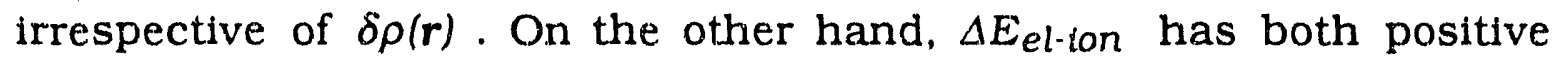
and negative contributions. Even though (A.5) is first-order in $\delta \rho(\boldsymbol{r})$, whereas (A.4) second-order, it should be emphasized that (A.5) vanishes for a partially filled outer shell in jellium, whereas (A.4) does not. The term (A.4) is, in fact, responsible for the ellipsoidal distortion $\mathbf{2 4}$ of jellium clusters for fillings other than electronic magic numbers.

The estimation of errors can therefore be made based on (A.4), which yields, for some simple choices of $\delta \rho(\boldsymbol{r})$ for a few values of $l(>0)$ and small $v$, and considering only non-magnetic states,

$$
\Delta E_{e l-e l} \sim 0.1\left(n / N^{2}\right) E_{e l \cdot e l}
$$




\section{F. References for chapter-II}

1. W. D. Knight, K. Clemenger, W. A. de Heer, W. A. Saunders, M. Y. Chou, and M. L. Cohen, Phys. Rev. Lett, 52, 2141 (1984).

2. For a detailed review and other references for earlier work see Walt A. de Heer, W. D. Knight, M. Y. Chou, and M. L. Cohen, Solid State Phys. 40, pp. 93-181 (1987).

3. H. Nishioka, K. Hansen, and B. R. Mottelson, to be published.

4. A. Rubio, L. C. Balbas, Ll. Serra, and M. Barranco, Phys. Rev. B 42 , 10950 (1990).

5. M. Brack, O. Genzken, K. Hansen, to appear in Phys. Rev. B.

6. O. Sugino, and H. Kamimura, Phys. Rev. Lett. 65, 2696 (1990).

7. S. W. Wang, L. M. Falicov, and A. W. Searcy, Surf. Sci. 143, 609 (1984).

8. W. D. Knight, W. A. de Heer, K. Clemenger, and W. A. Saunders, Solid State Comm. 53, 445 (1985).

9. W. D. Knight, K. Clemenger, W. A. de Heer, and W. A. Saunders, Phys. Rev. B 31, 2539 (1985). 
10. W. A. Saunders, K. Clemenger, W. A. de Heer, and W. D. Knight, Phys. Rev. B 32, 1366 (1985).

11. H. Göhlich, T. Lange, T. Bergmann and T. P. Martin, Phys. Rev. Lett. 65, 748 (1990).

12. T. P. Martin, T. Bergmann, H. Göhlich and T. Lange, Chem. Phys. Rev. Lett. 172, 209 (1990) and to be published.

13. M. L. Cohen, M. Y. Chou, W. D. Knight, and Walt A. de Heer, J. Phys. Chem. Solids 91, 3141 (1987).

14. W. Ekardt, Phys. Rev. B 29, 1558 (1984).

15. S. Bjфrnholm, J. Borggreen, O. Echt, K. Hansen, J. Pedersen, and H. D. Rasmussen, Phys. Rev. Lett. 65, 1627 (1990).

16. R. Kawai, and J. H. Weare, Phys. Rev. Lett. 65, 80 (1990) and references therein.

17. J. P. Bucher, P. Xia, and L. A. Bloomfield, Phys. Rev. B 42, 10858 (1990).

18. E. Engel, J. P. Perdew, Phys. Rev. B 43, 1331 (1991).

19. W. A. Harrison, Solid State Theory, Dover Publications Inc., New York (1979), pp. 440-443. 
20. A. Maiti, unpublished results.

21. The constant $\delta$ approximation is also supported by the fact that the total energy per atom did not change appreciably when tests were conducted with shifted values of $\delta$ around the chosen value.

22. E. G. Brovman, Y. Kagan, and A. Kholas, Sov. Phys. JETP 12, 786 (1970).

23. G. Apai, J. F. Hamilton, J. Stöhr, and A. Thomson, Phys. Rev. Lett. 43, 165 (1979).

24. K. Clemenger, Phys. Rev. B 32, 1359 (1985). 


\section{G. Tables for chapter-II}

Table 2.1

ENERGY F'ER ATOM FOR ELECTRONIC MAGIC NUMBERS

[ All energy components are in Rydbergs/Atom ]

\begin{tabular}{|c|c|c|c|c|c|c|}
\hline $\mathbf{N}$ & $\mathbf{E}_{\text {el-el }}$ & $\mathbf{E}_{\text {el-10n }}$ & $\mathbf{E}_{\text {lon-don }}$ & $\mathbf{E}_{\text {kdn }}$ & $\mathbf{E}_{\mathbf{x c}}$ & $\mathbf{E}_{\text {tot }}$ \\
\hline 8 & 1.2391 & -2.3050 & 0.8100 & 0.1699 & -0.2910 & -0.3770 \\
18 & 2.1018 & -4.1532 & 1.7793 & 0.1650 & -0.2950 & -0.4021 \\
20 & 2.2927 & -4.5329 & 1.9677 & 0.1636 & -0.2953 & -0.4042 \\
34 & 3.2017 & -6.3382 & 2.8527 & 0.1603 & -0.2973 & -0.4208 \\
40 & 3.6536 & -7.2733 & 3.3355 & 0.1603 & -0.2981 & -0.4220 \\
58 & 4.5780 & -9.1332 & 4.2724 & 0.1569 & -0.2988 & -0.4247 \\
68 & 5.1930 & -10.3257 & 4.8566 & 0.1581 & -0.2997 & -0.4177 \\
90 & 6.1319 & -12.2224 & 5.8122 & 0.1552 & -0.3001 & -0.4232 \\
92 & 6.2477 & -12.4628 & 5.9348 & 0.1546 & -0.3001 & -0.4258 \\
106 & 6.9693 & -13.8930 & 6.6458 & 0.1556 & -0.3009 & -0.4232 \\
132 & 7.9155 & -15.7607 & 7.5732 & 0.1537 & -0.3010 & -0.4193 \\
138 & 8.2188 & -16.3133 & 7.8164 & 0.1532 & -0.3011 & -0.4260 \\
168 & 9.2127 & -18.4019 & 8.9168 & 0.1539 & -0.3018 & -0.4203 \\
186 & 9.9586 & -19.8399 & 9.6047 & 0.1524 & -0.3018 & -0.4260 \\
196 & 10.4032 & -20.7524 & 10.0719 & 0.1523 & -0.3019 & -0.4269 \\
232 & 11.4760 & -22.9042 & 11.1480 & 0.1523 & -0.3024 & -0.4303 \\
\hline
\end{tabular}


Table 2.2

ENERGY PER ATOM FOR ATOMIC MAGIC NUMBBERS

[ Corresponding to finite bcc lattice clusters ]

\begin{tabular}{|c|c|c|c|c|c|c|}
\hline $\mathbf{N}$ & $\mathbf{E}_{\text {ei-el }}$ & $\mathbf{E}_{\text {el-1on }}$ & $\mathbf{E}_{\text {lon-10n }}$ & $\mathbf{E}_{\text {ldu }}$ & $\mathbf{E}_{x \mathbf{c}}$ & $\mathbf{E}_{\text {tot }}$ \\
\hline \hline \multirow{2}{*}{15} & 1.3347 & -2.4834 & 0.8942 & 0.1756 & -0.2906 & -0.3695 \\
27 & 1.8653 & -3.7496 & 1.6100 & 0.1725 & -0.2932 & -0.3950 \\
51 & 2.7594 & -5.5036 & 2.4774 & 0.1662 & -0.2956 & -0.3962 \\
59 & 4.2270 & -8.5267 & 4.0327 & 0.1600 & -0.2981 & -0.4051 \\
65 & 5.0171 & -10.0526 & 4.7625 & 0.1582 & -0.2992 & -0.4140 \\
89 & 6.0903 & -12.3516 & 5.9925 & 0.1555 & -0.3000 & -0.4133 \\
113 & 7.2265 & -14.6726 & 7.1688 & 0.1561 & -0.3007 & -0.4219 \\
137 & 8.1683 & -16.6656 & 8.2216 & 0.1533 & -0.3011 & -0.4235 \\
169 & 9.2546 & -18.4671 & 8.9386 & 0.1539 & -0.3018 & -0.4218 \\
181 & 9.7532 & -19.3720 & 9.3444 & 0.1529 & -0.3017 & -0.4232 \\
\hline
\end{tabular}


Table 2.3

ENERGY PER ATOM FOR ATOMIC MAGIC NUMBERS

[ corresponding to finite Type-I fcc lattice clusters]

\begin{tabular}{|c|c|c|c|c|c|c|}
\hline $\mathbf{N}$ & $\mathbf{E}_{\text {el-al }}$ & $\mathbf{E}_{\text {el-los }}$ & $\mathbf{E}_{\text {lom-10n }}$ & $\mathbf{E}_{\text {kin }}$ & $\mathbf{E}_{\mathbf{x c}}$ & $\mathbf{E}_{\text {tot }}$ \\
\hline \hline \multirow{2}{*}{13} & 1.6896 & -3.2914 & 1.3263 & 0.1766 & -0.2920 & -0.3909 \\
19 & 2.1970 & -4.3470 & 1.8778 & 0.1644 & -0.2947 & -0.4025 \\
43 & 3.8117 & -7.5802 & 3.4905 & 0.1611 & -0.2979 & -0.4148 \\
55 & 4.4281 & -8.8099 & 4.1072 & 0.1584 & -0.2985 & -0.4147 \\
79 & 5.6673 & -11.2833 & 5.3438 & 0.1577 & -0.2996 & -0.4141 \\
87 & 6.0062 & -11.9582 & 5.6743 & 0.1560 & -0.2999 & -0.4216 \\
135 & 8.0670 & -16.0499 & 7.7075 & 0.1535 & -0.3010 & -0.4229 \\
141 & 8.3192 & -16.6369 & 8.0351 & 0.1536 & -0.3011 & -0.4301 \\
177 & 9.5880 & -19.0662 & 9.1990 & 0.1533 & -0.3017 & -0.4276 \\
201 & 10.5821 & -21.1489 & 10.2932 & 0.1525 & -0.3020 & -0.4231 \\
& & & & & & \\
\hline
\end{tabular}


Table 2.4

ENERGY PER ATOM FOR ATOMIC MAGIC NUMBERS

[ corresponding to finite Type-II fcc lattice clusters]

\begin{tabular}{|c|c|c|c|c|c|c|}
\hline $\mathbf{N}$ & $\mathbf{E}_{\text {el-el }}$ & $\mathbf{E}_{\text {el-102 }}$ & $\mathbf{E}_{\text {ton-10n }}$ & $\mathbf{E}_{\mathbf{k l n}}$ & $\mathbf{E}_{\mathbf{x c}}$ & $\mathbf{E}_{\text {tot }}$ \\
\hline \hline \multirow{2}{*}{16} & 1.9409 & -3.8357 & 1.6303 & 0.1700 & -0.2938 & -0.3883 \\
28 & 2.8240 & -5.6009 & 2.5076 & 0.1656 & -0.2958 & -0.3995 \\
44 & 3.8641 & -7.6843 & 3.5448 & 0.1612 & -0.2978 & -0.4120 \\
68 & 5.1930 & -10.3257 & 4.8566 & 0.1581 & -0.2997 & -0.4177 \\
80 & 5.7100 & -11.2459 & 5.2609 & 0.1576 & -0.2996 & -0.4170 \\
104 & 6.8678 & -13.6798 & 6.5375 & 0.1561 & -0.3007 & -0.4191 \\
140 & 8.2857 & -16.5144 & 7.9477 & 0.1535 & -0.3011 & -0.4286 \\
152 & 8.6857 & -17.3414 & 8.3773 & 0.1543 & -0.3013 & -0.4254 \\
180 & 9.7120 & -19.3947 & 9.4122 & 0.1530 & -0.3017 & -0.4192 \\
\hline
\end{tabular}


Table 2.5

ENERGY PER ATOM FOR ATOMIC MAGIC NUMBERS

| corresponding to finite Type-III fcc lattice clusters|

\begin{tabular}{|c|c|c|c|c|c|c|}
\hline $\mathbf{N}$ & $\mathbf{E}_{\text {el-el }}$ & $\mathbf{E}_{\text {el-10n }}$ & $\mathbf{E}_{\text {lon-10n }}$ & $\mathbf{E}_{\text {Ldn }}$ & $\mathbf{E}_{\text {xe }}$ & $\mathbf{E}_{\text {tot }}$ \\
\hline \multirow{2}{*}{14} & 1.7748 & -3.4752 & 1.4315 & 0.1747 & -0.2926 & -0.3868 \\
38 & 3.5040 & -7.0220 & 3.2353 & 0.1607 & -0.2974 & -0.4194 \\
68 & 5.1930 & -10.3257 & 4.8566 & 0.1581 & -0.2997 & -0.4177 \\
92 & 6.2477 & -12.4628 & 5.9348 & 0.1546 & -0.3001 & -0.4258 \\
116 & 7.3363 & -14.6532 & 7.0340 & 0.1559 & -0.3007 & -0.4277 \\
164 & 9.0816 & -18.0950 & 8.7420 & 0.1541 & -0.3017 & -0.4190 \\
188 & 10.0476 & -20.0052 & 9.6792 & 0.1524 & -0.3018 & -0.4278 \\
236 & 11.6235 & -23.2000 & 11.2918 & 0.1522 & -0.3023 & -0.4348 \\
\hline
\end{tabular}




\section{H. Figures for chapter-II}

Figure 2.1

The effective pair-potential $V(r)$ (in milli Rydbergs) for bulk sodium as a function of radial distance $r$ (in units of Bohr radius $a_{B}$ ). It was obtained in the second order perturbation 19 of the local pseudopotential used. 


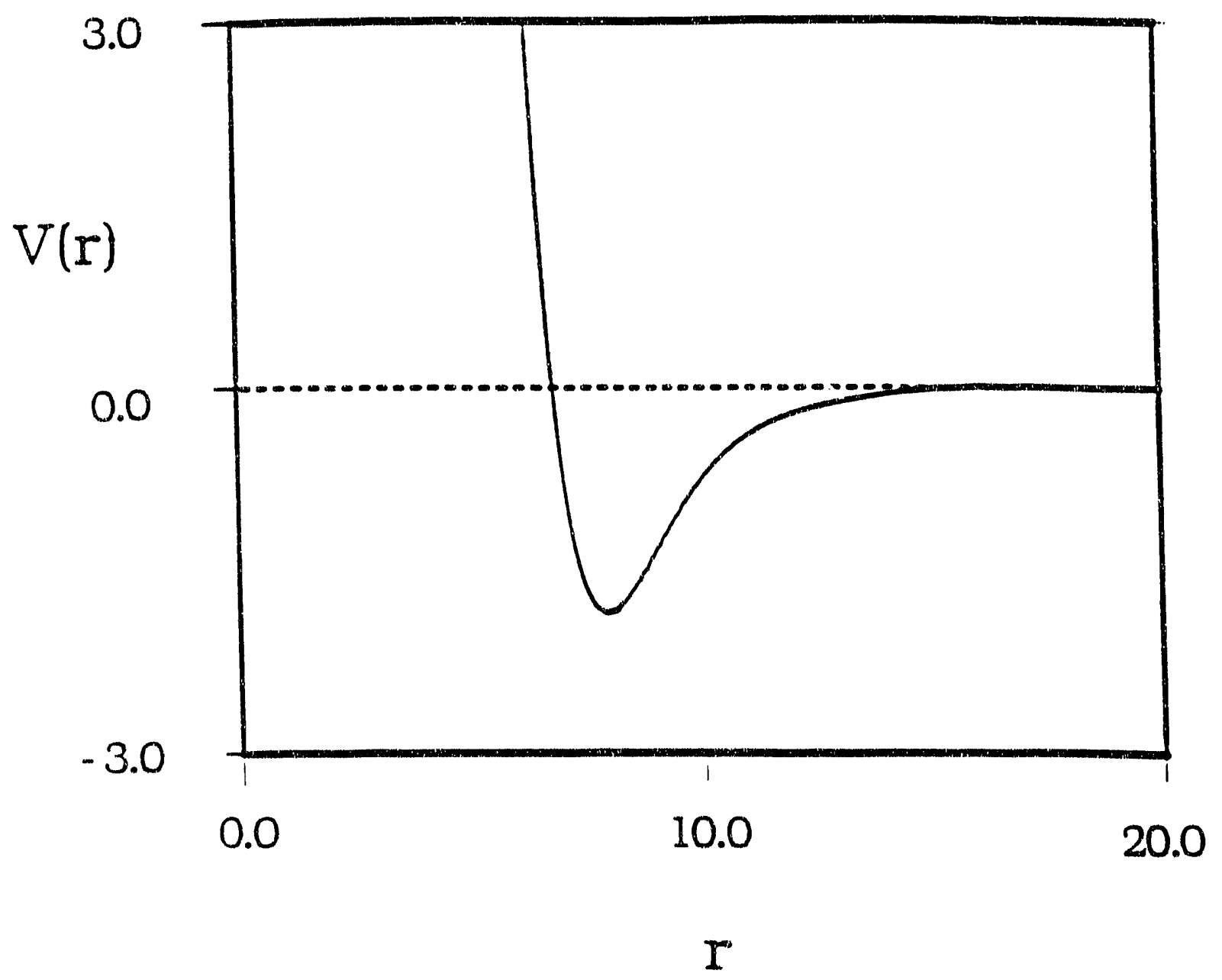


Figure 2.2

The approximate Kohn-sham potential used to confine the electrons in a cluster. The inner circle of radius $R_{+}$is a 2-D projection of the effective spherical jellium boundary, while the outer circle of radius $R$ represents the hard spherical wall. The difference in radii $\delta=R-R_{+}$is a measure of the characteristic decay-length through which the electronic charge spills out in a more realistic finite confining potential. 


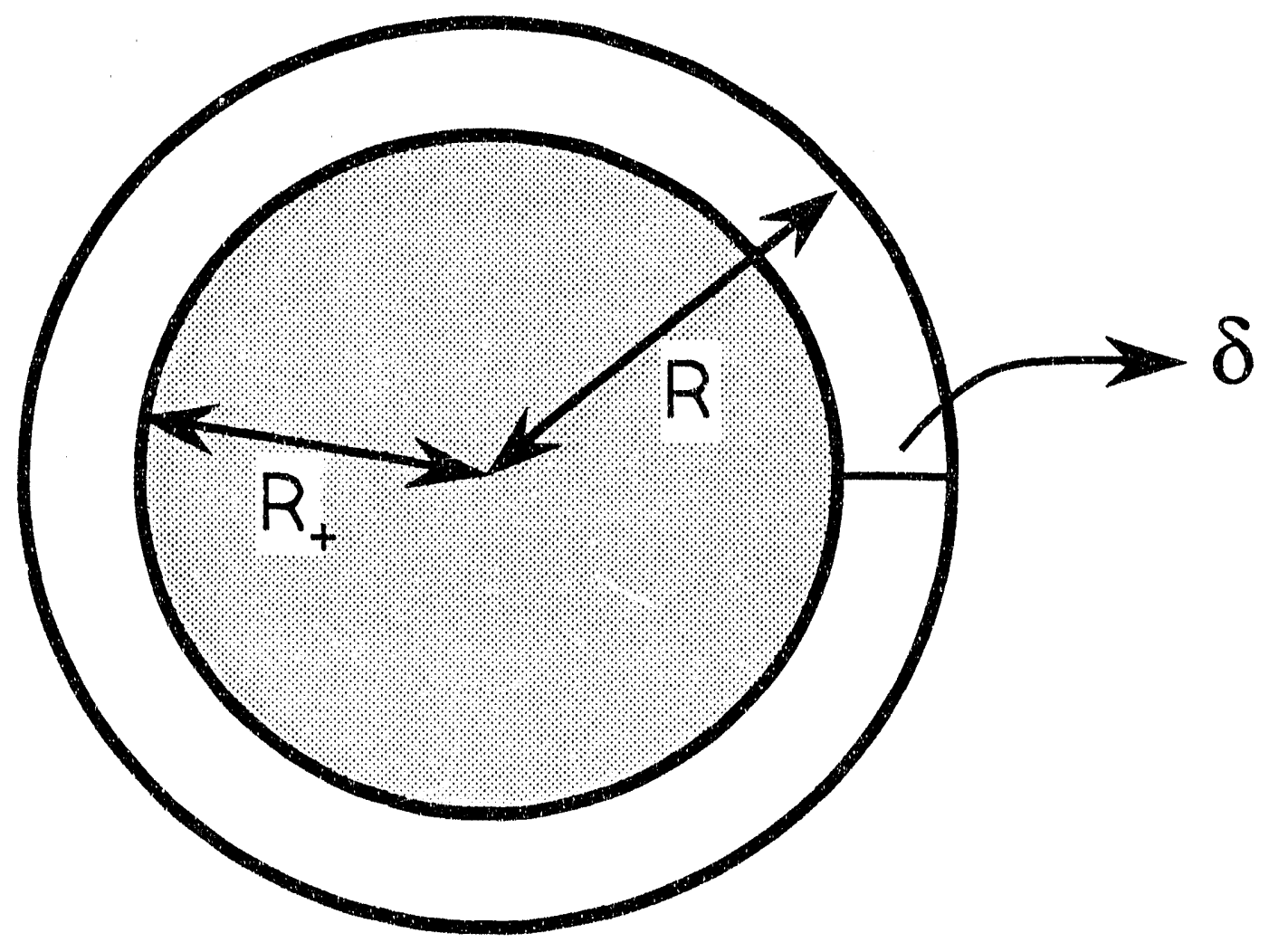


Figure 2.3

The electronic number density in the model under consideration for various electronic magic numbers as a function of the radial distance from the center of the spherical cluster. The number density is in units of the corresponding jellium density $\rho_{O}$, shown by the dashed line. The radial distance is in units of the total radius $R$ of the electron confining sphere (infinite potential well). (a) $\mathrm{N}=8$; (b) $\mathrm{N}=34$; (c) $\mathrm{N}=58$; (d) $\mathrm{N}=92$; and (e) $N=168$. 

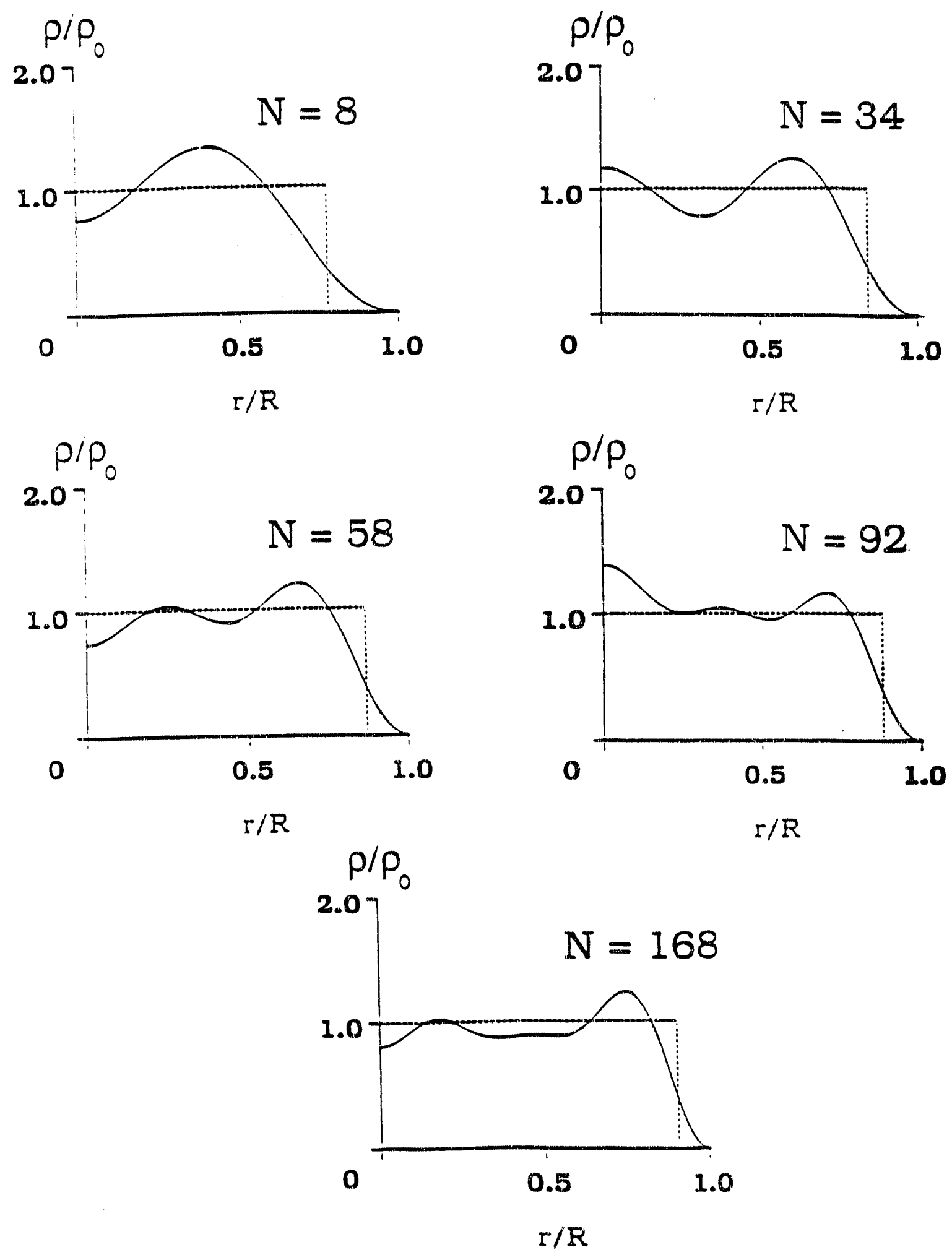
Figure 2.4

Plot of the total energy per atom as a function of cluster size $N$ for various electronic and atomic magic numbers (Tables 1 and 2). The crosses represent the points corresponding to the electronic magic numbers and the squares correspond to the atomic magic numbers. 


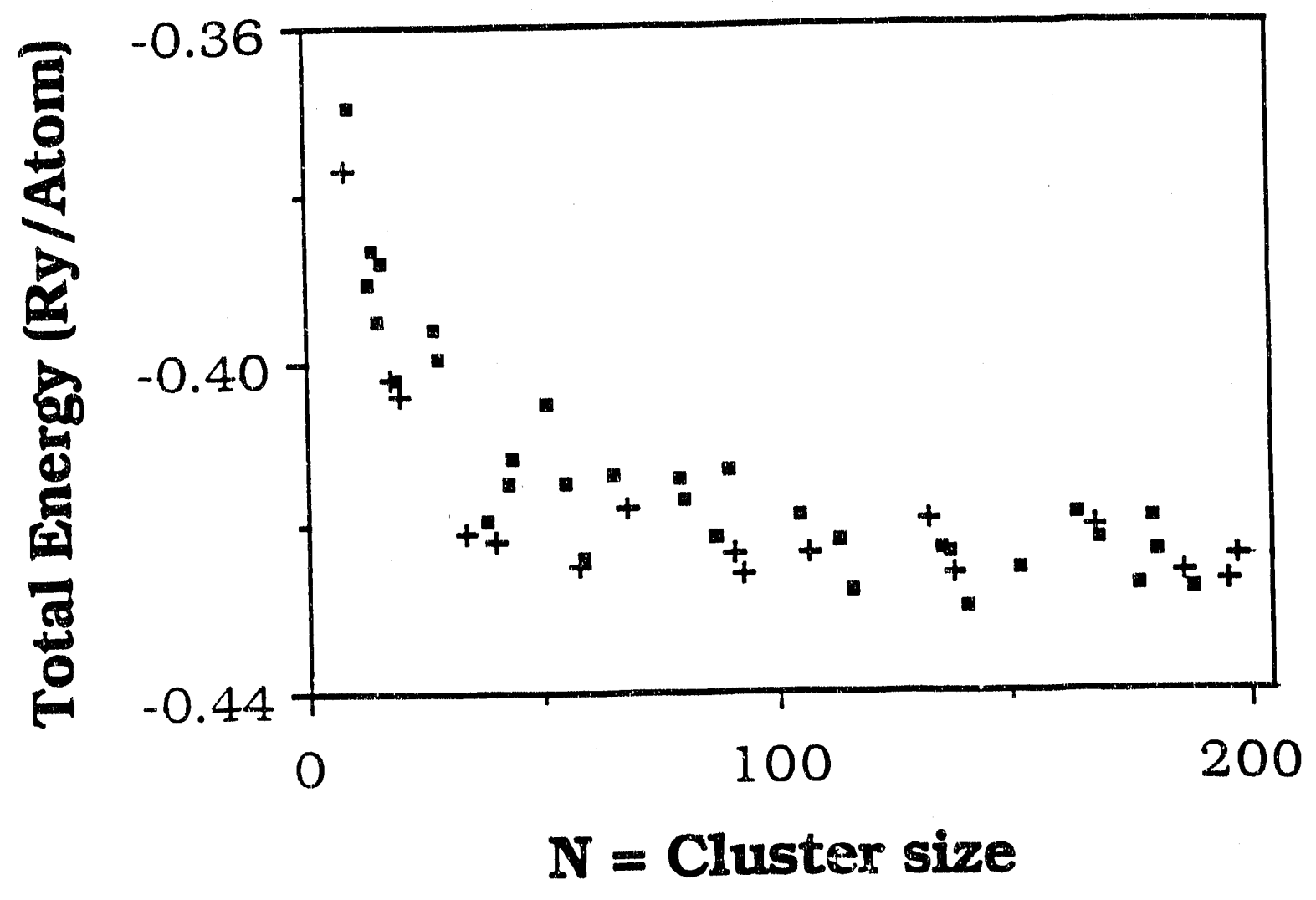


Figure 2.5

The convex "hulls" of minimum energy drawn separately for the electronic (crosses) and the atomic (squares) magic numbers, with a line joining them to form the overall minimum-energy hull. The clusters on this hull are the most stable ones. A clear transition in the nature of stability is observed between $N=58$ (electronic magic number) and $N=141$ (atomic magic number). 


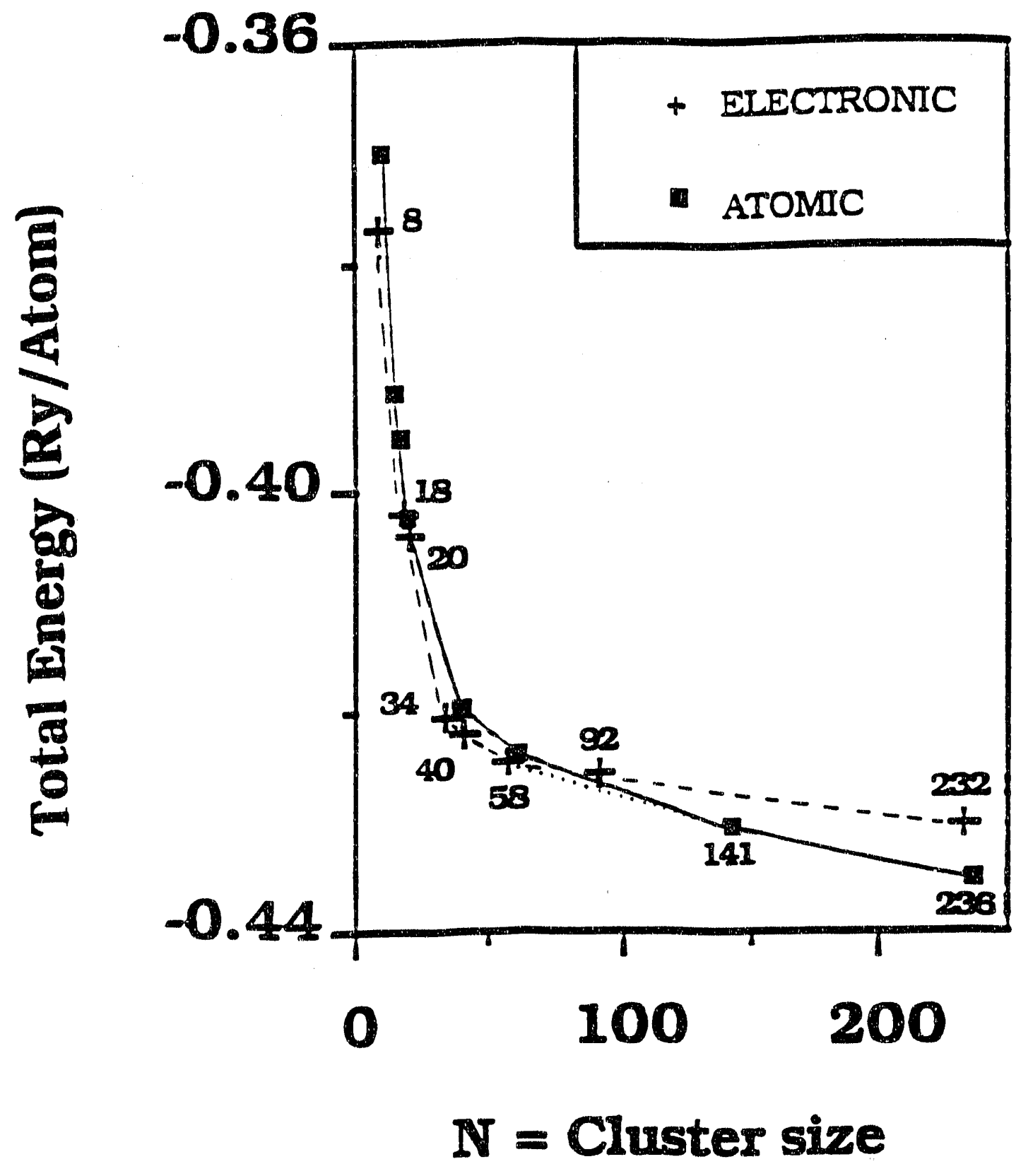




\section{Chapter-III. Phase diagram for small sodium clusters}

\section{A. Introduction}

The zero-temperature calculations of chapter II clearly show a transition of cluster stability from "electronic" magic numbers at small sizes to geometrically closed "atomic" magic numbers at sizes larger than a critical value. This critical value of $N$, estimated to be $\sim 100$, is much lower than the value $\sim 1,500$ where the cross-over described above had been experimentally established by Martin 1,2. Moreover, Bjørnholm's group in Denmark, at experimental temperatures presumably higher than that of Martin's, did not observe geometrically closed stable structures ${ }^{3}$; based on theoretical estimates 4-6 $^{-6}$ they expected no atomic structural effects even for clusters as large as several thousand. The abundance spectra in the two expcriments differed also for cluster sizes less than a thousand -- both in the sharpness of features and in the exact location of the electronic magic numbers ${ }^{2,3}$. Martin's data, for instance, had many more "spikes" i.e. local maxima at sizes that cannot be interpreted as electronic shellclosings. At the same time, the stable electronic magic numbers in the two spectra differ significantly, notwithstanding finite limits of sizeresolution in the two experiments. It is clear that finite temperature, neglected in the calculation of chapter-II, plays an important role, and consideration of finite temperature effects is essential in addressing the discrepancies discussed above. The model used in chapter II is superior to molecular-dynamics or similar "classical" calculations 7-12 in this regard, not only because of the much less computational effort involved, but also because it is inherently a quantum mechanical 
calculation. This model, for instance, is able to describe quantum phenomena such as zero-point oscillations of the ions that cannot be, in principle, estimated in a molecular-dynamics simulation. It is important to note here, however, that the dynamical details of cluster formation, growth and fragmentation might and, in fact, do influence the experimentally observed cluster stability. Unfortunately, these complicated dynamic processes lie well beyond the realm of calculational techniques described in this thesis, and perfect thermal equilibrium has been assumed on the time scale of all experiments.

The present chapter extends the calculations of chapter II to larger clusters $(200<N<800)$, and finite temperatures. It confirms the earlier results at $T=0: f c c$ lattice-induced (close-packed) clusters are the stable phase in this size regime. For finite temperatures a new picture emerges: at "high temperatures" the experimentally observed stable clusters are to be interpreted as solely caused by thermal melting of the clusters. Jellium becomes an appropriate starting point for total-energy calculations at temperatures beyond melting. For a large range of intermediate temperatures (i.e. below the cluster melting point) bcc lattice-induced structures appear to be the most stable ones, as is seen in the schematic phase diagram reported below.

A new physical interpretation of the electronic magic numbers, where cluster stability is observed in high-temperature experiments. is also obtained in terms of an enhanced uniformity of calculated electronic charge density for these particularly stable cluster sizes.

\section{B. The madel}

The model system and the hamiltonian for total energy 
calculation are described in detail in the previous chapter. The starting point for any given cluster size $N$ is to place the ionic cores at regular finite lattice sites. Density functional theory is used and the effective Kohn-Sham potential seen by each electron is approximated by a flat potential well bounded by an infinite wall, the shape of which is, in principle, determined by the position of the outermost atoms of the cluster. The finite lattice is then relaxed in a "rigid" electronic background to get to the ionic structure corresponding to the lowest energy configuration. Only wells of spherical shapes are considered because of practical, calculational requirements. For concreteness, bcc (bulk crystalline structure of metallic sodium above $5 \mathrm{~K}$ ) and $f c c$ (representative of a close-packed structure) lattices truncated by the electron-confining sphere are used as starting-point structures for the clusters. In conformity with the high (spherical) symmetry of the electron environment, truncated lattices of only tetrahedral or higher point-group symmetry are included. The electronic charge outside the jellium sphere, caused by the finiteness of the cluster work-function. is taken into account by placing the effective uniform jellium sphere a "decay-length" $\delta=0.29 a$ inside the electron confining wall, where $a$ is the bulk lattice-constant for $\mathrm{Na}$. The energy involved in the distortion of the electronic charge density at and around the ionic sites is calculated in the first-order perturbation of the local pseudopotential; exchange and correlation effects are handled in the local-density approximation (LDA) scheme ${ }^{13}$. The total energy consists of electronic kinetic energy, exchange-correlation energy, the firstorder pseudopotential contribution, and the electrostatic energy. The last contribution is a sum of three terms: the electron-electron 
Coulomb repulsion, the ion-ion Madelung energy, and the electron-ion Coulomb attraction. For any given cluster size, the spherical shells of atoms are radially and angularly relaxed to reach the lowest energy configuration compatible with the chosen spherical symmetry of the electronic charge.

\section{Calculations and Results}

In chapter II it is shown that when a zero-temperature total energy hull is constructed for all cluster sizes $N$, the nature of the most stable clusters, i.e., those on the hull, changes as a function of $N$. For smaller sizes the clusters on the hull owe their stability to quantal effects and correspond to complete electronic angular-momentum shells. Such clusters comprise a series of so-called electronic magic numbers. The larger clusters, on the other hand, always belong $t / s$ an $f c c$ cluster truncated by the electron-confining sphere, with cornplete spherical shells of atoms. Such clusters form a series of geometricallyclosed $f c c$ lattice-induced numbers. The largest of the electronic magic-number series and the smallest of the $f c c$ series on the totalenergy hull are 58 and 141 respectively, i.e., the dividing line between the two series is approximately at $N \sim 100$. Calculations for $N>200$ show that the electronic magic numbers are always less stable, at $T=$ 0 , than the structures with complete atomic shells. Figure 3.1 shows the total energy hull, at $T=0$, of the lattice-induced geometricallyclosed structures only. All clusters on this hull (solid line) are found to belong to an $f c c$ (close-packed) series. In view of the finitetemperature effects discussed below, a separate hull (dashed line) is also drawn for the geometrically closed $b c c$ series. Some of the 
clusters very close to the hulls in energy are also shown. Fits to the two hulls (at $T=0$ ) as a function of cluster size $N$ in the asymptotic form ${ }^{14}$ are given by:

$$
\begin{aligned}
& E_{f c c}[R y / \text { atom }]=-0.45804+0.10486 n+0.22905 n^{2}, \\
& E_{b c c}[R y / \text { atom }]=-0.45770+0.10516 n+0.33005 n^{2},
\end{aligned}
$$

where $n=N^{-1 / 3}$, and the results for total energy per atom in the bulk $(n=0) f c c$ and $b c c$ crystalline phases of sodium have been used 13,15.

In order to obtain the amplitude of lattice vibrations at arbitrary temperatures the vibrational frequency spectrum is approximated by the angular modes of oscillation of the complete spherical shells of atoms. The melting point of a cluster is determined by applying the Lindemann criterion to this shear mode, i.e., to the angular oscillation of an atom on the outermost shell: the lattice melts if the amplitude of such oscillations relative to the layer below is $10 \%$ or more of the distance to the nearest neighbor. All other modes (e.g., radial) or displacements (e.g., inner shells) are stiffer, corresponding to smaller displacements and not contributing substantially to the melting of the cluster ${ }^{\mathbf{1 6}}$. The melting temperatures $T_{M}(N)$ thus calculated for several cluster sizes are : $T_{M}(51)=T_{M}(65)=0 \mathrm{~K}$; $T_{M}(89)=158 \mathrm{~K} ; \quad T_{M}(137)=253 \mathrm{~K} ; T_{M}(259)=285 \mathrm{~K} ; T_{M}(411)$ $=317 \mathrm{~K}$. The known result $T_{M}(\infty)=372 \mathrm{~K}$ has also been used to ensure proper asymptotic limit.

It should be noted that $T_{M}=0$ for $N<65$, i.e., the melting is produced by the zero-point vibrations for the smallest clusters, a pure quantum-mechanical effect; $T_{M}$ increases sharply between $N \sim 65$ and 
$N \sim 200$. For larger $N$ it rises slowly, with a few oscillations, and it reaches asymptotically the bulk value of $T_{M}=372 \mathrm{~K}$ as $N$ approaches infinity. Figure 3.2 shows an interpolated melting curve (with the oscillations removed) as the lower bound of the phase termed "jellium". Physically, the higher polarizability of smaller clusters ${ }^{17}$ implies larger screening, lower oscillation frequencies, and larger lattice vibrations at a given temperature: hence lower melting points. For $N<65$, the zero-point motion of the ionic cores is large enough to cause lattice melting, and jellium becomes a good model even at $T=0$. For $N>65$, however, the stability at the electronic magic numbers predicted from SJBM calculations should be observed only at temperatures above the melting curve. In the experiments of Ref. 4 . the cluster temperatures have been estimated to be $\sim 400-500 \mathrm{~K}$, clearly above the melting curve of Fig. 3.2. Another important feature of Fig. 3.2 is the existence of a large region just below the melting curve where the geometrically-closed $b c c$ lattice-induced structures become energetically favorable. This result is in agreement with the Landau theory of solidification 18,19 .

For the curve separating the $b c c$ and $f c c$ (close-packed) phases in Fig. 3.2, one notes that the difference in free energy between the two phases, as a function of temperature $T$ and $n=N^{-1 / 3}$ is given by $\Delta F(n, T)=\Delta U(n, T)-T . \Delta S(n, T)$, where $\Delta U$ and $\Delta S$ are the corresponding differences in internal energy and entropy per atom respectively. Calculations using the shear and radial frequency modes show conclusively that for all $n$, the difference in internal energy $\Delta U$ does not change significantly with temperature. This result, coupled with the assumption that $\Delta S$ does not strongly depend on $T$ and $n$ 
yields the temperature at which a transition from one structure to the other takes place. Such a cross-over temperature, obtained by solving the equation $\Delta F(n, T)=0$ is given by $T_{\text {cross-over }}(n)=\Delta U(n, 0) / \Delta S(0,0)$. The entropy difference $\Delta S(0,0)$ between the two phases for the bulk at zero temperature is estimated from the temperature $T \sim 5 \mathrm{~K}$ at which bulk sodium has a martensitic transformation from $h c p$ to $b c c$. and the known fact that both close-packed structures, hcp and $f c c$. are very close in energy 15. Therefore this fcc-bcc crossover temperature as a function of $n$ is given by:

$$
T_{\text {cross-over }}(n)=\frac{E_{b c c}(n)-E_{f c c}(n)}{E_{b c c}(0)-E_{f c c}(0)} \cdot 5 \mathrm{~K}
$$

where $E_{b c c}(n)$ and $E_{f c c}(n)$ are given by Eqns. (3.1) and (3.2) respectively.

The existence of the bcc phase is well supported by the experimental results of Ref. 2, where the cluster temperatures are lower than those of Ref. 3, presumably close to the melting curve of Fig. 3.2. The maxima in the abundance spectrum of Ref. 2, although interpreted as electronic magic numbers, are all consistently shifted away from the magic numbers of Ref. 3 towards the bcc magic numbers of the present calculation, i.e. those on the dashed hull of Fig. 3.1. Also the lower temperature data exhibit local stability at perhaps other types of geometrically closed structures not considered here.

A new physical interpretation of the experimentally observed electronic magic numbers is obtained by plotting the quantity, 


$$
\Delta \rho=\operatorname{Min}_{<\rho>} \int_{|\rho(r)-<\rho>| 4 \pi r^{2} d r}
$$

as a function of cluster size between 200 and 800 for $N$ with complete electronic angular-momentum shells (Fig. 3.3). In (3.4) above, $\rho(r)$ is the electronic number density normalized in terms of the uniform jellium density; $\langle\rho\rangle$ is a constant density that minimizes $\Delta \rho$. If a hull for $\Delta \rho$ is constructed, one obtains a series of cluster sizes at which the electronic charge density is more uniform. These numbers, shown in Fig. 3.3 are exactly the ones identified as the electronic magic numbers in uniform SJBM calculations 2,3 . Physically, uniform jellium does the most efficient job of electrostatic charge cancellation in these particular clusters, with a lowering of the electrostatic energy and enhancement of cluster stability. This result lends a strong support to the notion that these experimentally observed jellium clusters at high temperatures are "liquidlike" clusters, where relatively rapid ionic motion gives rise to an effective continuous positive background rather than a static distribution of sodium-ion potentials.

\section{Conclusion}

The total energy calculation of sodium clusters based on the model described in chapter II has been extended to clusters of larger sizes, up to $N \sim 800$. The calculation performed at $T=0$ emphasizes once again the result that for $N>100$ geometrically closed clusters are more stable than the ones with closed electronic angular-momentum shells. Moreover, geometrically closed $f c c$ (close-packed) structures turn out to have lower energies than the geometrically closed $b c c$ clusters. Two separate energy hulls have been contructed for the most 
stable $f c c$ and $b c c$ clusters and large $N$ asymptotic fits to each hull indicates no cross-over at a finite $N$. This result is supported by the existence of a stable close-packed phase of bulk sodium below $5 \mathrm{~K}$, and use has been made of this experimental fact to determine a $b c c-f c c$ cross-over curve as a function of cluster-size.

To investigate the nature (i.e. whether "solidlike" or "liquidlike") of any cluster at a given temperature, the low-lying angular (shear) modes of the outermost shell have been considered. Statistical mechanics has been used to compute the mean square displacement of a given atom on the outer shell, and the Lindernann criterion to determine whether the cluster is "solidlike" or "liquidlike". Such a calculation enables one to determine the melting point as a function of cluster size $N$. It is found that for $N<65$ atoms have large zero-point motion, and clusters are "liquidlike" even at $T=0$. For $N>65$, the melting temperature increases on the whole as a function of $N$ with a few oscillations. It is to be recalled from results in chapter II that in the region $N<65$ electronic magic numbers are the stable clusters at $T=0$, and these are also the same magic numbers obtained from calculations based on uniform jellium models. The stable jellium magic number clusters observed experimentally as peaks in the abundance spectra are, therefore, to be identified as liquidlike clusters. The existence of a $b c c$ phase just below the melting curve is in accord with trends in shift of the abundance spectrum peaks with temperature.

Finally, the electronic charge density is found to be the most uniform for clusters corresponding to some electronic magic numbers viz. precisely the ones obtained as energetically stable uniform jellium 
clusters. This fact is consistent with the picture depicted above : the observed stable electronic magic number clusters are "liquidlike" clusters indeed, where a time-averaged rapid ionic motion effectively mimics a uniform jellium background, that cancels out the uniform electronic charge density to lower the electrostatic energy substantially. 


\section{E. References for chapter-III}

1. T. P. Martin, T. Bergmann, H. Göhlich, and T, Lange, Chem. Phys. Lett. 172, 209 (1990), Z. Phys. D 19, 25 (1991).

2. H. Göhlich, T. Lange, T. Bergmann, and T. P. Martin, Phys. Rev. Lett. 65, 748 (1990); Mod. Phys. Lett. 5, 101 (1991); Z. Phys. D 19, 117 (1991).

3. S. Bjфrnholm, J. Borggreen, O. Echt, K. Hansen, J. Pedersen, and H. D. Rasmussen, Phys. Rev. Lett. 65, 1627 (1990); Z. Phys. D 19. 47 (1991).

4. T. Lange, H. Göhlich, T. Bergmann, and T. P. Martin, Z. Phys. D 19, 113 (1991).

5. T. Bergmann, and T. P. Martin, J. Chem. Phys. 90, 2848 (1989).

6. H. Nishioka, K. Hansen, and B. R. Mottelson, Phys. Rev. B 42, 9377 (1990).

7. R. Car, and M. Parrinello, Phys. Rev. Lett. 55, 2471 (1985).

8. P. Ballone, W. Andreoni, R. Car, and M. Parrinello, Europhys. Lett. 8, 73 (1989).

9. R. Kawai, J. H. Weare, Phys. Rev. Lett. 65, 80 (1990). 
10. O. Sugino, and H. Kamimura, Phys. Rev. Lett. 65, 2696 (1990).

11. O. B. Christensen, K. W. Jacobsen, J. K. Nфrskov, and M. Manninen, Phys. Rev. Lett. 66, 2219 (1991).

12. J. Y. Yi, J. O. Dirk, and J. Bernholc, Phys. Rev. Lett. 67, 1594 (1991).

13. E. G. Brovman, Y. Kagan, and A. Kholas, Fiz. Tuerd. Tela 12, 1001 (1970) [Sov. Phys. Solid State 12, 786 (1970)].

14. E. Engel, and J. P. Perdew, Phys. Rev. B 43, 1331 (1991).

15. W. A. Harrison, Pseudopotentials in the theory of metals, (Benjamin, New York, 1966), p. 193.

16. The temperature effects on the electronic structure -. considered by M. Brack, O. Genzken, and K. Hansen, Z. Phys. D 19, 51 (1991) and by U. Gupta, and A. K. Rajagopal, Phys. Rep. 87, 259 (1982) -are considerably smaller than those studied here, and therefore not included.

17. W. A. de Heer, W. D. Knight, M. Y. Chou, and M. L. Cohen, in Solid State Physics, edited by H. Ehrenreich, F. Seitz, and D. Turnbull (Academic, New York, 1987), vol. 40, pp. 93-181. 
18. S. Alexander, and J. McTague, Phys. Rev. Lett. 41, 702 (1978).

19. Icosahedral-symmetry structures, which could have a lower free energy near the melting curve (Ref. 22), because of the considerable faceting do not fit in a spherical-charge scheme. Icosahedral clusters are therefore not considered here. It is known that they do not exist for small clusters, $N<1000$ at the experimental temperatures (Ref. 3). For $N>1000$, however, faceted structures are known to become important, and over an intermediate range of cluster sizes and a given range of temperatures, they should become stable. They have probably been observed (Ref. 5). There should be therefore modifications in the phase diagram for temperatures close to $T_{M}$ and for $N>1000$. 


\section{F. Figures for chapter-III}

Figure 3.1

Total energy hull at $T=0$ (solid line) for the geometrically closed lattice-induced structures for cluster sizes $200<N<800$. Only $f c c$ latticeinduced structures denoted by black squares are on this hull. The most stable geometrically closed $b c c$ clusters (denoted by ' + 's) and the corresponding hull (dashed line) are indicated. Clusters very close to the hulls are also shown. 


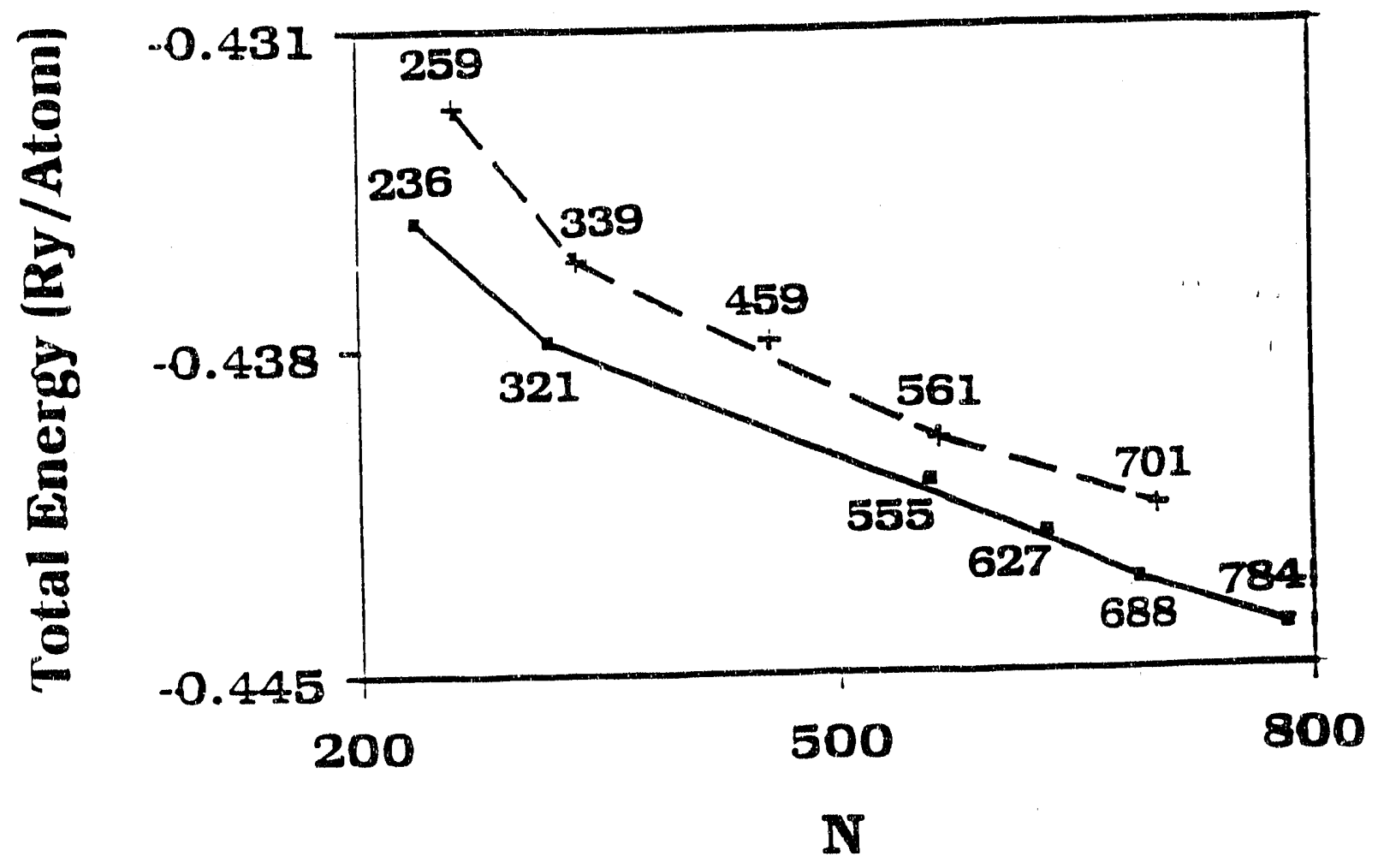


Figure 3.2

Temperature-size phase diagram for clusters of size $N<1000$. The monotonically increasing curve separating the "jellium" and "bcc" phases is the "interpolated" melting curve of sodium clusters as a function of size $N$, with finer oscillatory features removed. It intersects the $\mathrm{N}$ axis at $\mathrm{N} \sim 65$. The bcc-fcc cross-over curve is obtained from equation (3.3). 


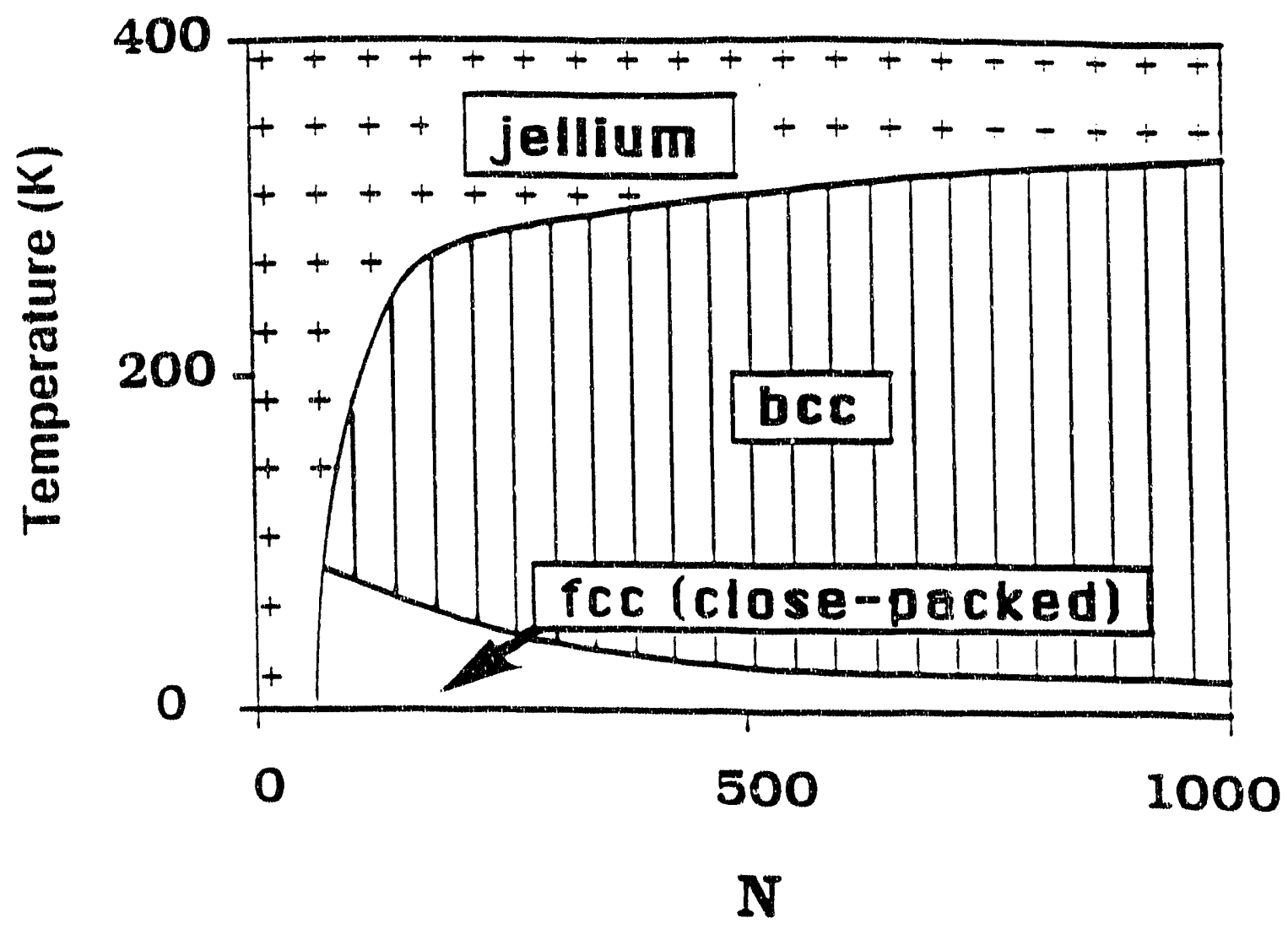


Figure 3.3

Non-uniformity in electron density $\Delta \rho$ (defined in the text), in arbitrary units, for clusters with complete electronic angular-momentum shells. Cluster sizes on the stability hull are indicated by the proper number labels. It is to be noted that these numbers are all "electronic" magic numbers. 


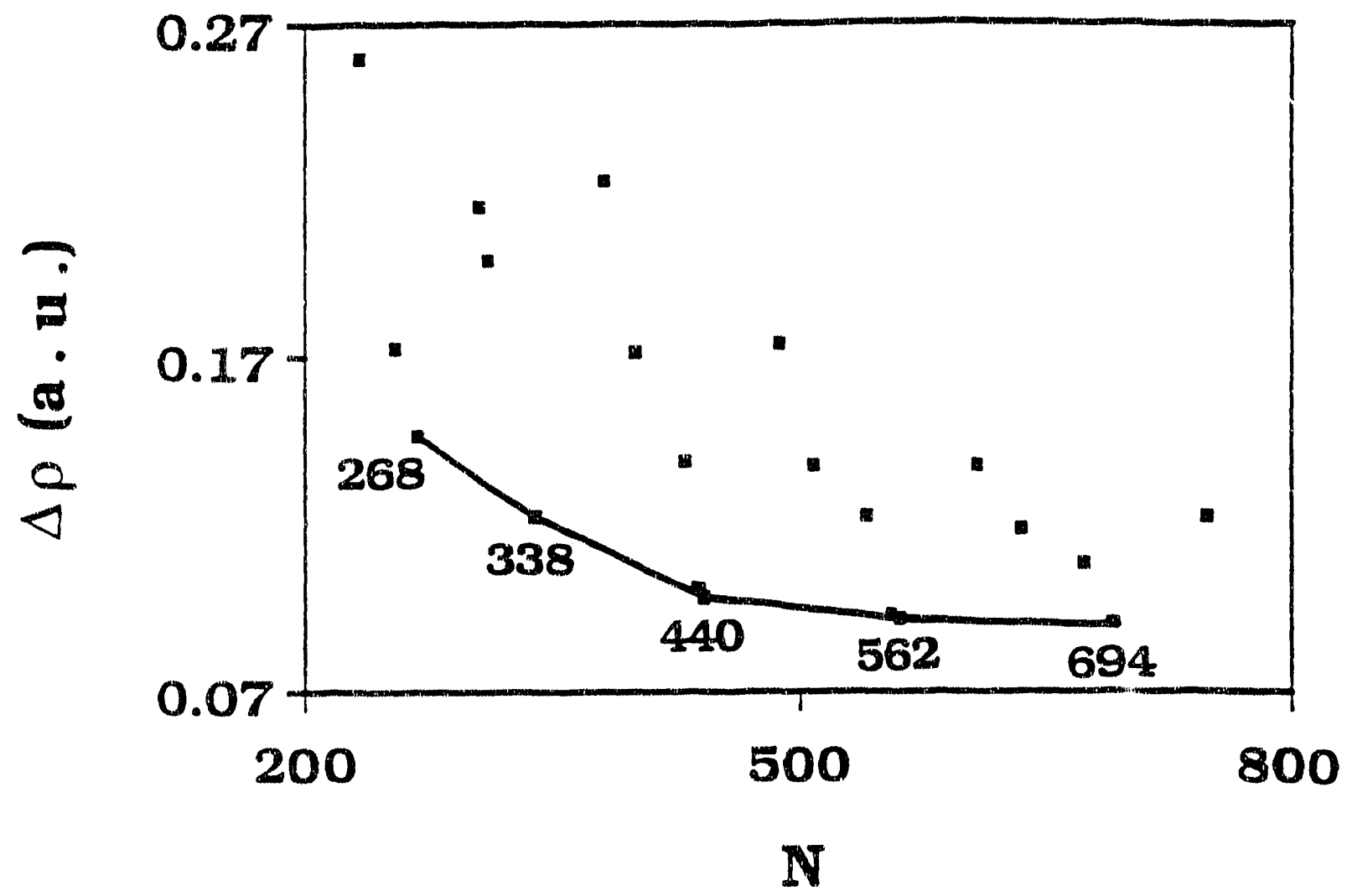




\section{Chapter- IV : Local chemical potential approach to small cluster many"}

\section{body systems}

\section{A. Introduction}

Since its introduction ${ }^{1}$ in 1963 the Hubbard model has been frequently used in the literature to investigate a whole range of manybody effects: ferromagnetism, antiferromagnetism, metal-insulator transition, charge-density waves and spin-density waves to name only a few 1-6. The model has been applied to a variety of lattices of different dimensions $2,3.7$ and general theorems have been proved in some cases 8 . Unfortunately, no three-dimensional macroscopic Hubbard system is susceptible to exact treatment. However, considerable insight into macroscopic systems can be obtained from the exact solution of small subsystems, which, though not expected to unfold the whole story, can sometimes provide important clues to the problem. This exact small-cluster approach has been used successfully in situations where local many-body effects are important: clusters of size two to eight ${ }^{\mathbf{9 - 2 5}}$, photoemission ${ }^{13}$ behavior in $\mathrm{Ni}$, intermediatevalence 14,15 behavior in $\mathrm{Ce}$, magnetic behavior ${ }^{16}$ in $\mathrm{Fe}$, alloying in $\mathrm{Cu}$ Au system 17, many-body effects in a heavy-fermion system 18, thermodynamic properties 19 , valence-bond formation 20 as well as in understanding of the two-dimensional (2D) electronic properties in the $\mathrm{Cu}-\mathrm{O}$ planes of high-temperature superconductors 21 .

This chapter focuses on the study of properties of a small open subsystem of the cluster, one that exchanges particles with the rest of the system, in order to infer the behavior of the global properties of 
the system as a whole. In particular, an attempt is made to extract physical properties out of the analysis of the quantum mechanical particle number fluctuation in the subsystem as a function of various parameters. Exact calculations are performed on two systems: a fouratom linear chain with periodic boundary conditions 26 and a fouratom tetrahedral cluster with periodic boundary conditions, the smallest non-trivial $f c c$ cluster. The hamiltonian chosen is the Hubbard model with hopping matrix element $t>0$ between nearest neighbors only and an on-site interaction $U$, which can be repulsive or attractive:

$$
H_{O}=-t \sum_{<i j>\sigma}\left(c_{i b} c_{j \sigma}+c_{j \sigma}^{\dagger} c_{i \sigma}\right)+U \sum_{i} \hat{n}_{i \uparrow} \hat{n}_{i \downarrow} .
$$

where $i, j$ are the atomic site indices, $<\ldots>$ indicates nearest-neighbor pairs and $\sigma$ is the spin index. For notational simplicity, henceforth, it is convenient to choose $|t|$ as the unit of energy and express $H_{O}, U$ and temperature $T$ in dimensionless form : $H=H_{0} /|t|, x=U /|t|$ and $\tau=k_{B} T /|t|$ respectively, where $k_{B}$ is Boltzmann's constant. Thus the hamiltonian can be recast in the form:

$$
H=\sum_{<i j>\sigma}\left(c_{i} \neq c_{j \sigma}+c_{j} t c_{i \sigma}\right)+x \sum_{i} \hat{n}_{i \uparrow} \hat{n}_{i \downarrow} .
$$

Since the many-body correlations are short-range in the Hubbard model, it is interesting to study the local particle-number fluctuation $\Delta$ at a particular site, labelled $\alpha$, as a function of $n, x$ and $\tau$. Here $n$, the average number of electrons at the $\alpha$-site, is given by 
$<\left\langle\hat{n}>>\right.$, the ensemble average of $\hat{n}=\hat{n}_{\alpha \uparrow}+\hat{n}_{\alpha \downarrow}$, the particle number operator at the relevant site. The local particle fluctuation $\Delta$ is defined as :

$$
\Delta=\Delta(n, x, \tau)=<\hat{n}^{2} \gg-<<\hat{\imath}>2 .
$$

The generalized grand canonical ensemble average of any observable $\hat{A}$ is defined by :

$$
<\left\langle\hat{A}>>=\frac{\operatorname{Tr}[\hat{A} \exp [-(H-M \hat{N}-\mu \hat{A}) / \tau\}]}{\operatorname{Tr}[\exp \{-(H-M \hat{N}-\mu \hat{n}) / \tau\}]}\right.
$$

where $M$ is the chemical potential associated with the total particle number operator $\hat{N}=\Sigma_{i} \hat{n}_{l}$ in the system, and $\mu$ is an 'extra' local chemical potential associated with the $\alpha$-site, the usefulness of which is discussed below. In these expressions, the subscript $i$ in the summation over $\hat{n}_{t}$ runs over $N_{O}$ sites, where $N_{O}$ is the total number of sites in the system.

It should be noted that the ordinary grand canonical ensemble corresponds to the particular value $\mu=0$. In that particular case the averages shown in (4.3) reduce, for $\tau \rightarrow 0$, to averages over expectation values in the ground states of $(4.1 \mathrm{~b})$ belonging to various total number of electrons, $N$, which is the eigenvalue of $\hat{N}$, and takes only integral values from $O$ to $2 N_{O}$. To determine which ground states of given integral $N$ values do actually contribute to this average, the groundstate energy eigenvalues of $(4.1 \mathrm{~b})$ should be plotted as a function of the integer $N$. A "hull" is then constructed by joining these points in pairs such that all points lie either on the "hull" or above it. Only the ground 
states for those integral number of electrons that lie on the hull do contribute to the averages in (4.3); ground states which are above the hull do not contribute. For example if the ground states for $N=2, N$ $=3$, and $N=4$, are such that

$$
E_{G}(N=3)>\frac{1}{2}\left\{E_{G}(N=2)+E_{G}(N=4)\right\} .
$$

then the contribution to (4.3) for $\mu=0, \tau \rightarrow 0$ and for the average total particle number $<<\hat{N} \gg>$ (integral or fractional, including $<\langle\hat{N}\rangle>=2$,

$<\langle\hat{N}\rangle>=3$, and $\langle<\hat{N}\rangle>=4$,) in the range $2 \leq<\langle\hat{N}>>\leq 4$, arise solely from the ground states of $N=2$ and $N=4$, without participation of the $N=3$ ground state.

In the ensemble with $\mu=O, M$ is a monotonically increasing function of $n$, as is required by the chemical stability of the system. In view of the discussion to follow, it is to be noted that a discontinutty in the chernical potential $M$ at a given value $n=n^{*}$, i.e.,

$$
M_{+}-M_{-}>0 \text {, }
$$

where $M_{+}$and $M_{-}$are the values of $M$ as $n^{*}$ is approached respectively from the positive and negative sides, characterizes, by definition, a Mott insulator at the occupation $n^{*}$.

\section{B. Calculations and results}

In order to calculate the local particle-number fluctuation $\Delta$ for general values of $n$ and $x$, one has to take recourse to numerical calculations. To perform the ensemble averages (4.3) it is convenient to work in the basis of the eigenstates of the generalized hamiltonian 
$H^{\prime}=(H-M \hat{N}-\mu \hat{\imath})$. Reduction in the size of the matrices to be diagonalized can be achieved by taking note of the following quantities that commute with the generalized hamiltonian $H^{\prime}$ :

(i) $\hat{N}$, the total number operator;

(ii) $S_{z}$, the $z$-component of the total spin angular-momentum;

(iii) $\mathbf{S}^{2}$, the total spin operator; and

(iv) the permutation operator of some site-indices.

For convenience, the calculations reported here have made use of the constants of motion (i), (ii) and (iv) only, which reduces the original problem of diagonalizing a $256 \times 256$ matrix to ones of size no greater than $18 \times 18$. Exact analytical results, however, are obtained for certain limiting values of $n, x$ and $\tau$. Thus, one has the following results :

$$
\begin{aligned}
& \Delta(n, x=0, \tau)=n(2-n) / 2 ; \\
& \Delta(n, x \rightarrow \infty, \tau)=\begin{array}{l}
n(1-n), \\
(n-1)(2-n), \quad \begin{array}{c}
0 \leq n \leq 1, \\
1 \leq n \leq 2 ;
\end{array}
\end{array} \\
& \Delta(n, x \rightarrow-\infty, \tau)=n(2-n) ; \\
& \Delta(n, x, \tau \rightarrow \infty)=n(2-n) / 2 .
\end{aligned}
$$

It is also straightforward to show that, for bipartite 27 lattices $\Delta$ is symmetric about $n=1$, i.e.,

$$
\Delta(n, x, \tau)=\Delta(2-n, x, \tau)
$$

Equation (4.9) holds for the four-atom ring, but not in general for the 
tetrahedral cluster, because $f c c$ is not a bipartite lattice 27 . It should be noted here that $\Delta$ for a fully spin-polarized loosely speaking ferromagnetic) state, for all interaction strengths, assume the value equal to that of the $x \rightarrow \infty$ limit as given by equation $(4.6)$.

In the rest of the chapter, attention is confined only to the zero temperature $(\tau \rightarrow 0)$ properties in order to deal with some of the difficulties involved in extrapolating small-cluster results to the thermodynamic limit at low temperatures. The difficulties arise from the discreteness of the energy spectra of finite clusters. In particular, use is made of the additional local chemical potential $\mu$ to distinguish between the 'genuine' singularities in $\Delta$ (those that survive as the number of sites is increased up to the thermodynamic limit) and the 'spurious' singularities (those that are present only because of the finiteness of the cluster).

In the following, use is made of a zero-temperature identity that relates the local particle-number fluctuation to a derivative of the ground-state energy :

$$
\Delta(n, x, \tau=0)=n(1-n)+2 \frac{\partial E_{G}(n, x)}{\partial x} .
$$

where $E_{G}(n, x)$ is the ground state energy per site corresponding to the occupation $n$. This result is proven in Appendix-I of this chapter. It is to be noted that when $\mu=0$, i.e., in the exact grand canonical ensemble average, all sites are equivalent, so that $n=\langle\langle\hat{A}\rangle\rangle / N_{O}$ is the fractional occupation, i.e., the number of electrons per site. An important result to study the Mott insulator state is obtained by taking the derivative of $(4.10)$ with respect to $n$ and then the difference 
between values obtained by approaching the point of discontinuity from the positive and the negative sides; this procedure yields:

$$
\left(\frac{\partial \Delta}{\partial n}\right)_{+}-\left(\frac{\partial \Delta}{\partial r}\right)_{-}=2 \frac{\partial}{\partial x}\left(M_{+}-M_{-}\right)
$$

where $M_{ \pm}=\left(\partial E_{G}(n, x) / \partial n\right)_{ \pm}$, corresponding to the partial derivatives on the right and left respectively. Equation (4.11) establishes a direct relationship between the discontinuity in the slope of $\Delta$ with that of the chemical potential $M$ (both sides of (4.11) are zero in case of no discontinuity). Analyticity as a function of $x$ has been assumed, as $\left(M_{+}-M_{-}\right)$is found to change smoothly with $x$, except at $x=0$ and at other isolated points.

Let us concentrate first on an analysis of the two small-cluster systems in the exact grand canonical ensemble $(\mu=0)$. Figures 4.1 and 4.2 display graphical tabulations of the eigenvalues $N$ of the total particle number operator that contribute to the relevant ground states at each point in the $n-x$ plane, for the ring and the tetrahedral cluster respectively when the interaction is repulsive $(x>0)$. Figure 4.3 gives the corresponding description in the attractive interaction $(x<0)$ case for either system. The solid black lines correspond to the values where ground states belong to the subspace of a single $N$. The region in between are 'mixed' domains where relevant ground states belong to subspaces of more than one $N$. Several important results associated with the above structure follow :

(i) Results for $x \rightarrow 0$ and $x=0$ are different for finite-cluster systems and approach each other only in the thermodynamic limit. This 
discontinuity arises from the presence of several 'accidental degeneracies' for the particular value $x=0$. The singular $x=0$ line is, therefore, omitted in Figs. 4.1, 2 and 3.

(ii) The local particle fluctuation is a continuous function of $n$ with a continuous partial derivative $\partial \Delta / \partial n$ and a negative second derivative $\partial^{2} \Delta / \partial n^{2}$ for all regions of phase-space except at the solid black (single $N$ ) lines.

(iii) On the solid black lines, where a single $N$ value contributes, the local particle fluctuation $\Delta(x, n)$ exhibit discontinuities in $\partial \Delta / \partial n$ (kinks) as a function of $n$ (for fixed $x$ ) with the vertex pointing either upwards or downwards. On these lines, the chemical potential takes a finite range of values $M_{N}(\min ) \leq M \leq M_{N}(\max )$ and, when plotted as a function of $M$, kinks in $\Delta(x, M)$ occur at $M=M_{N}(\min )$ and $M=M_{N}(\max )$.

(iv) Ground states belonging to all $N$ from $O$ to $2 N_{O}$ are not, in general, sampled as $n$ increases from $O$ to 2 for a fixed $x$. No odd $N$. for instance, contributes to the ground states for an attractive interaction $(x<0)$ for either system. Also for a repulsive interaction $(x>0)$, the $N=3$ and $N=5$ states do not contribute for $x<4.6$ in case of the four-atom ring whereas the $N=3$ state does not contribute for any $x>0$ in the tetrahedral cluster ${ }^{28}$.

(v) In a white region, where more than a single $N$ contribute, only the two $N$ values corresponding to the two solid black lines bordering the relevant white region contribute in general. For specific models and/or specific values of the parameters, however, it is possible to have extra accidental degeneracies, where more than two values of $N$ contribute to the ground state. [Such case can 
be seen, for example, in the tetrahedral cluster for $1.25<n<2$ for all $x>0$.]

For finite clusters, only two behaviors (i.e. negative second derivative in a white region and kinks on the solid black lines) of $\Delta(x, n)$ are found, as discussed above.

As the number of sites $N_{O}$ increases, the structures of Figs. 4.1, 2 and 3 may change in a number of ways to lead to the following possible scenarios in the limit $N_{O} \rightarrow \infty$ :

(1) The number of solid black lines might increase with the attendant rearrangement of the mixed- $N$ contribution and, in the thermodynamic limit, the $n-x$ plane may consist of a continuous plane of single $N$ contributions, i.e., a "black" plane.

(2) The number of solid black lines might increase but remain a discrete set; in the thermodynamic limit the $n-x$ plane would still look similar to Figs. 4.1, 2 and 3, but possibly with many more solid black lines separated by "white"(mixed $M$ ) areas.

(3) In general it is expected in the thermodynamic limit $N_{O} \rightarrow \infty$, that, the $n-x$ plane will exhibit regions of

(3a) continuous $N$ values ("black" areas)

(3b) continuous admixture of two $N$ values ("white" areas)

(3c) isolated lines of single $N$ values (solid black lines)

(3d) perhaps regions of dense but discrete black lines (e.g. all rational values of $n$ allowed, all irrational ones not present ${ }^{22}$ ).

In the thermodynamic limit $\left(N_{O} \rightarrow \infty\right)$, in a "white" region, where more than one $N$ contribute, $\Delta$ is always a continuous function, 
with a continuous $\partial \Delta / \partial n$, and with a negative second derivative $\partial^{2} \Delta / \partial n^{2}$. This property is proven in Appendix-II. In a "black" area or line, where a single $N$ contributes, there is a possibility of $\Delta$ behaving differently: either it may develop positive second derivatives with respect to $n$, discontinuities in $\partial \Delta / \partial n$, -- i.e., kinks -- at discrete values of $n$ or possibly other types of pathological behavior. Not all structures are physically possible, and each requires individual analysis. A kink, however, by necessity arises from the discontinuity in the slope, which along with (4.11) implies a Mott insulator. Other singular structures may be characteristic of other phases for the values $\left(n^{*}, x^{*}\right)$ where they appear.

In the finite clusters under consideration here, the discontinuities in $\partial \Delta / \partial n$ occur only on the solid black lines. This behavior is shown in Figures 4.4 and 4.5. The discontinuities correspond to a single $N$ at a particular value of $n$, labelled $n^{*}$. It is along these lines that the "local" chemical potential $\mu$ can be effectively used to test the stability of the single- $N$ structures and to distinguish between kinks that are "genuine" and those that are "spurious" -- i.e., induced by the finiteness of the cluster. The "local" chemical potential $\mu$ allows a local variation of $n$ at the site in question, whereas the value of $N$ in the cluster as a whole remains constant. This procedure "opens up" the $\alpha$-site to be less dependent on its environment, which in this case is the small cluster. This partial decoupling emphasizes the local aspects of the many-body problem and may shed light into the behavior of the system in the thermodynamic limit, when the environment becomes macroscopically large. 
The resulting analysis is valid only in "single- $N$ " regions, i.e., along the isolated black lines, or in a black region, -- a finite interval of $n$ around $n=n^{*}$-- if that is the case in the thermodynamic limit. The analysis therefore, in the finite clusters, is carried out not only for the discrete values of $n$ where a single $N$ contributes, but also in their immediate neighborhoods.

The working assumption is that, by looking at the behavior of $\Delta$ as a function of $n$, as $\mu$ is varied from a small negative to a small positive value, one can decide whether the structure of $\partial \Delta / \partial n$ at that occupation $n$ is genuine or just an effect of the finiteness of the cluster. The result of this analysis may produce the following situations in the thermodynamic limit :

(A) The curve $\Delta(n)$ takes at $n=n^{*}$ one of the limiting forms described in (4.5)-(4.7); in particular the form (4.6) is taken for the ferromagnetic case. In such a situation, the exact grand canonical ensemble $(\mu=0)$ itself yields the appropriate limiting form at the occupations in question.

(B) There is a positive second derivative of $\Delta$ with respect to $n$ at $n=n^{*}$; this is a strong indication of instability, with the formation, in the thermodynamic limit, of an energy gap in the spectrum, i.e., a real kink, as shown in (4.11).

(C) If $\Delta$ exhibits a negative second derivative with respect to $n$ several possibilities other than case (A) arise.

(C1) Even though the second derivative with respect to $n$ is negative at $n=n^{*}$. it becomes positive for values of $n$ reasonably close to $n^{*}$.

(C2) The second derivative with respect to $n$ is negative everywhere 
in the immediate vicinity of $n=n^{*}$.

Case (A) should be interpreted as a possible magnetic phase at $n=n^{*}$; case $(C 1)$ as a tendency to the formation of a Mott insulator state - - in the thermodynamic limit -- at a value of $n$ close or even equal to $n^{*}$; case (C2) as a "spurious" singularity, i.e., a consequence of the finiteness of the cluster.

Figures 4.6 and 4.7 display the variation of $\Delta$ with $n$ on and around each of the solid black lines for the four-atom ring and the tetrahedral cluster respectively, for various fixed values of $x$ and at zero temperature. Both attractive $(x<0)$ and repulsive $(x>0)$ values of the interaction are considered. The case $x>0$ is more interesting and is considered first. For the four-atom ring the following features are worth noticing :

(i) The curve $\Delta$ versus $n$ has positive second derivative at and around $n=1$ (i.e. half-filled band) for all values of $x>0$ (case (B)). This indicates a Mott insulator at that concentration, setting in at arbitrarily small repulsive interactions, in agreement with the results of Lieb and $\mathrm{Wu}^{7}$. Because of its isomorphism 26,29 to lattices in $2 \mathrm{D}$ and $3 \mathrm{D}$, this result seems to indicate a Mott transition for all $x>0$ at $n=1$ for all bipartite lattices 27 in one, two or three dimensions.

It is interesting to note that if the second-nearest neighbor hopping is included, the positive second derivative does not appear at $n=1$, for small positive values of $x$, but oniy in a small neighborhood of $n=1$, i.e., case $(C 1)$. Whether that happens for $n>1$ or $n<1$ depends on the sign of the second-nearest neighbor hopping element relative to the first-nearest neighbor hopping 
parameter. As $x$ increases, the interval of positive second derivative grows, and covers the $n=1$ point i.e., case (B). In any case, the formation of a Mott insulator at the $n=1$ concentration for all $x>0$ is clearly indicated by the test.

(ii) The $N=3 \quad(n=0.75)$ and $N=5 \quad(n=1.25)$ cases exhibit a transition to a ferromagnetic state as a function of $x$ for $x=x^{*}=18.5$. For $\mu=0$. i.e., in the exact grand canonical ensemble, as $x$ increases the local particle fluctuation $\Delta$ discontinuously drops from a value 0.1946 for $x<x^{*}$ to the value 0.1875 given by equation (II.2) for $x>x^{*}$ and remains constant for any greater value of $x$.

(iii) The curve has negative second derivative at and around all other solid black lines. They all belong to the case (C2) discussed above except for $N=1$ and $N=7$.

(iv) For $N=1$ and $N=7, \Delta$ takes the limiting form given by (II.2) [ case (A) ]. The reason is a trivial transition to a spin-polarized behavior when a single carrier (electron or hole) exists in the finite lattice. For larger clusters this property appears at $n=1 / N_{O}$ and $n=2-1 / N_{O}$ which, in the thermodynamic limit, are the empty $n=0$ and fully occupied $n=2$ lattices.

From the analysis of the four-atom ring, it is possible to state that in the seven partial occupations where Mott-insulator singularities may occur for the infinite one-dimensional chain or the perfect square lattice, only $n=1$ should exhibit insulating behavior for all $x>0$. This behavior agrees with the Monte Carlo calculations for the infinite chain and the $2 D$ square lattice 30,31 .

The tetrahedral cluster has a more complicated and interesting structure. The main features are : 
(i) The structure at $n=1$ falls in the category (C1) discussed above. For infinitesimal $x(>0)$ a small portion of the curve has positive second derivative at $n \sim 1.05$. As $x$ increases, the interval of positive second derivative gradually grows, and includes the $n=1$ point for $x>1.2$. This result should be considered as a signature of a Mott insulator occurring at $n=1$ for arbitrarily small positive values of $x$. Interestingly, this behavior at $n=1$ is similar to that of the fouratom ring with positive second-nearest-neighbor hopping: when the second nearest-neighbor hopping parameter in the ring is equal to the nearest-neighbor hopping parameter, one obtains a system topologically identical to the tetrahedral cluster.

(ii) There is a transition to a ferromagnetic state for all $x>0$ as a function of $n$ at $n=1.25 \quad(N=5)$. The curve around the solid black line at this occupation assumes the limiting form of (4.6). This behavior is not observed for the ring with second nearest neighbor hopping $(\because t)$ and seems to be intrinsic to the tetrahedral cluster and possibly, in the thermodynamic limit, to the fcc lattice of which the tetrahedral cluster is the basic building block.

(iii) The curve $\Delta(n)$ has the form (4.6) in the full interval $1.25 \leq n \leq$ 2. This behavior is related to the accidental degeneracies shown in Fig 4.2.

(iv) There is the small-cluster induced spin-polarization behavior at $N=1 \quad(n=0.25)$ and $N=7 \quad(n=1.75)$, characteristic of all four-atom clusters.

In order to ascertain whether a spin-polarized (ferromagnetic) state exists at a given occupation in the thermodynamic limit, one could use an exiension of the analysis with the local chemical potential 
$\mu$. Thus, for instance, one could increase $n$ from $n=1$ by increasing $\mu$ to the proper value corresponding to $n=1.25$ while staying within the $N=4$ subspace. One then looks for a 'flow' of the ground state from a spin-'unpolarized' state at $n=1$ to a fully spin-polarized (ferromagnetic) state at $n=1.25$, and as a result whether $\Delta$ at $n=1.25$ assumes a value given by (4.6). The result holds for the tetrahedral cluster, indicating that the ferromagnetic transition in the tetrahedral cluster might survive in the thermodynamic limit possibly at an occupation close to $n=1.25$. On the other hand, similar analysis at $n=0.25(N=1)$, by changing the occupation from $n=0.5$ down to $n=0.25$ by decreasing $\mu$ while remaining within the $N=2$ subspace 33 shows that the ground state does not 'flow' into a spin-polarized state at $n=0.25$ indicating that the transition to a magnetic state is 'spurious', i.e., a consequence of the finite number of sites in the cluster. Similar analysis by decreasing $n$ from $n=1$ to $n=0.75$ while remaining within the $N=4$ subspace for $x>18.5$ for the four-atom ring does not also result in a 'flow' into spin-polarized state. This implies that in the thermodynamic limit, this transition to a fully spin-polarized (ferromagnetic) state does not occur at $n=0.75$ (or 1.25) but at $N=N_{O} \pm 1$ in accordance with the results of Nagaoka ${ }^{34}$.

For an attractive interaction $x<0$, on the other hand, the $\Delta-n$ curve always has a negative second derivative everywhere in the vicinity of each solid black line (case (C2)), implying that there are no Mott insulators or magnetized states. The only interesting feature is the absence of the $N=$ odd values for either system at all values of $x<0$. This result indicates a tendency of the electrons to pair, which might imply superconductivity (Cooper pairing) or physically bound 
bielectrons, which might lead to Bose-condensation.

\section{Discussion}

By thtroducing a local chemical potential at a single site of a small cluster, the local occupation of that site can be allowed to vary with respect to the average site-occupation of the cluster as a whole. This partial decoupling of one site from the rest should be more sensitive to the local environment of the particular site than to the overall size and shape of the cluster.

This technique has been applied to clusters with varying occupation of particles, after the analysis of particular global states has been completed. Since the smallness of the cluster allows only a finite number of average occupations, the method proposed here introduces additional continuity into an essentially discrete system. It is thus possible to analyze differential properties of occupations, and analyze the local stability of particular solutions, distinguishing behaviors which are 'genuine' properties of the system as a whole (those that might survive in the thermodynamic limit) from those 'spurious' properties which are caused exclusively by the finiteness of the cluster.

Two examples were analyzed. Both are Hubbard models in fouratom clusters with different connectivities : a four-atom ring (representative of the infinite chain, 2D square lattice and the $b c c$ lattice) and a tetrahedral cluster (representative of the $f c c$ structure). The following results were obtained :

(1) The $n=1$ half-filled band exhibits for all cases 'genuine' Mott- 
insulating states for all repulsive interactions.

(2) In bipartite lattices, the inclusion of an arbitrarily small secondnearest neighbor hopping seems to decrease the stability of the Mott-insulating state, although no supression has been observed.

(3) The intervals $0 \leq n \leq 0.25\left(0 \leq n \leq 1 / N_{O}\right.$ for an arbitrary cluster of size $\left.N_{0}\right)$ and $1.75 \leq n \leq 2\left(2-1 / N_{0} \leq n \leq 2\right)$ yield trivial uninteresting result corresponding to a single electron or a single hole. The states are always fully spin-polarized and may or may not represent the behavior in the thermodynamic limit.

(4) The tetrahedral cluster exhibits a ferromagnetic state in the interval $1.25 \leq n \leq 2$, which remains stable under the test of the flow of states with changing local chemical potential $\mu$.

(5) The four-atom ring exhibits a ferromagnetic state at $n=0.75$ and $n=1.25$ in the exact grand canonical ensemble $(\mu=0)$ for $x>18.5$. Such state at the above fractional occupations is the well-known Nagaoka ferromagnetic state at $N=N_{O} \pm 1$, and is not stable under the applied variable $\mu$ test; in the thermodynamic limit, in accordance with Nagaoka's theorem ${ }^{34}$ this feeble ferromagnet could only occur at $n=1$, i.e., only (exactly) at $N=N_{O} \pm 1$.

(6) Attractive interactions lead to 'genuine' instabilities of odd $N$ number states and 'genuine' stable conditions for even $N$. No magnetic solutions or Mott insulators appear in this case. Indication of electron pairing (i.e., either Cooper pairs or bielectrons) is clearly evident. 


\section{Appendix-I to chapter-IV}

Proof of equation (4.10).

$$
\begin{aligned}
A^{2} & =\left(\hat{A}_{\alpha \uparrow}+\hat{A}_{\alpha \downarrow}\right)^{2} \\
& =\hat{n}+2 \hat{n}_{\alpha \uparrow} \hat{n}_{\alpha \downarrow},
\end{aligned}
$$

because $\hat{A}_{\alpha \uparrow}$ and $\hat{n}_{\alpha \downarrow}$ are projection operators, e.g. $\left(A_{\alpha \uparrow}\right)^{2}=\hat{n}_{\alpha \uparrow}$. Thus (I.2) yields :

$$
\Delta=\left\langle\left\langle\hat{n}^{2}\right\rangle\right\rangle \cdot n^{2}=n(1-n)+2\left\langle\left\langle\hat{n}_{\alpha \uparrow} \hat{n}_{\alpha \downarrow}\right\rangle\right\rangle .
$$

At $\tau=0$,

$$
\begin{aligned}
N_{O} E_{G}=\langle H\rangle & =\left\langle H_{T}\right\rangle+x \sum_{i}\left\langle\hat{n}_{i \uparrow} \hat{H}_{i \downarrow}\right\rangle, \\
& =\left\langle H_{T}\right\rangle+x N_{O}\left\langle\hat{n}_{\alpha \uparrow} \hat{H}_{\alpha \downarrow}\right\rangle,
\end{aligned}
$$

where use has been made of the assumption that all the $N_{O}$ sites have identical occupations and the ground state does not have any spontaneously broken symmetry, i.e., there are no spin-density waves or spiral spin arrangements. In the equation above, $\langle\ldots\rangle$ denotes the quantum mechanical expectation in the ground state, $H_{T}$ is the (nearest neighbor) hopping part of the hamiltonian $H$ and is independent of interaction parameter $x$. Use of the Feynman-Hellman theorem on (AI.2) together with (AI.1) at zero temperature proves result $(4.10)$. 


\section{E. Appendix-II to chapter-IV}

This appendix proves that in a 'white' region (ignoring the special case of accidental degeneracies) $\Delta$ has a negative second derivative with respect to $n$. For a given value of $x$, let $n$ - and $n_{+}(n$.< $\left.n_{+}\right)$be the occupations containing the solid black lines that border the 'white' region in question to the left and right respectively. An arbitrary occupation $n$, and the local particle fluctuation $\Delta$ at $n$ inside the white region (i.e., $n_{-}<n<n_{+}$) are given by the interpolation formulas :

$$
\begin{aligned}
n & =\lambda n_{-}+(1-\lambda) n_{+}: \\
\Delta & \left.=\lambda<\left\langle\hat{n}^{2}\right\rangle\right\rangle_{-}+(1-\lambda)<\left\langle\hat{n}^{2}\right\rangle_{+}-n^{2}, \\
& =\lambda\left(\Delta_{-}+n_{-}^{2}\right)+(1-\lambda)\left(\Delta_{+}+n_{+} 2\right)-n^{2},
\end{aligned}
$$

where subscripts - and + refer to averages on the solid black lines at $n_{-}$and $n_{+}$respectively. Inversion of (AII.1) and use of that result in (AII.2) yields,

$$
\Delta=\frac{n-n_{-}}{n_{+}-n_{-}}\left(\Delta-n_{-}^{2}\right)+\frac{n_{+} \cdot n}{n_{+}-n_{-}}\left(\Delta_{+}+n_{+} 2\right)-n^{2}
$$

The first two terms on the RHS of (AII.3) are linear in $n$ and, therefore,

$$
\frac{\partial^{2} \Delta}{\partial n^{2}}=-2<0
$$

which is the result quoted. 


\section{F. References for chapter-IV}

1. J. Hubbard, Proc. R. Soc. London, Ser. A276, 238 (1963); 227, 237 (1964); 281, 401 (1964); 285, 542 (1965); 296, 100 (1967).

2. D. R. Penn, Phys. Rev. 142, 350 (1966).

3. D.Denley and L. M. Falicov, Phys. Rev. B17, 1289 (1978).

4. D. Adler, in Solid State Physics, ed. by H. Ehrenreich, F. Seitz and D. Turnbull (Academic, New York, 1968), vol.21, p.1.

5. Proceedings of the International Conference on Metal-nonmetal Transitions, San Francisco, 1968 [Rev. Mod. Phys. 40.673 (1968)].

6. N. F. Mott and Z. Zimmerman, Rep. Prog. Phys. 33, 881 (1970).

7. E. H. Lieb and F. Y. Wu, Phys. Rev. Lett. 20, 1445 (1968).

8. E. H. Lieb and D. Mattis, Phys. Rev. 125, 164 (1962).

9. H. Shiba and P. A. Pincus, Phys. Rev. B5, 1966 (1972).

10. H. Shiba, Prog. Theor. Phys., 48, 6B, 2171 (1972). 
11. L. M. Falicov and R. A. Harris, J. Chem. Phys. 51, 3153 (1969).

12. L. M. Falicov and R. H. Victora, Phys, Rev. B 30, 1695 (1984).

13. R. H. Victora and L. M. Falicov, Phys. Rev. Lett. 55, 1140 (1985).

14. A. Reich and L. M. Falicov, Phys, Rev. B34, 6752 (1986).

15. J. C. Parlebas, R. H. Victora and L. M. Falicov, J. Phys. (Paris) 47, 1029 (1986).

16. E. C. Sowa and L. M. Falicov, Phys. Rev. B35, 3765 (1987).

17. A. Reich and L. M. Falicov, Phys. Rev. B36, 3117 1987).

18. A. Reich and L. M. Falicov, Phys. Rev. B37, 5560 (1988).

19. J. Callaway, D. P. Chen and R. Tang, Z. Phys. D3, 91 (1986); Phys. Rev. B35, 3705 (1987).

20. J. Callaway, Phys. Rev. B35, 8723 (1987).

21. M. Ogata and H. Shiba, J. Phys. Soc. Jpn. 57, 9. 3074 (1988).

22. J. K. Freericks and L. M. Falicov, Phys. Rev. B41, 2163 (1990).

23. D. J. Newman, K. S. Chan, and B. Ng, J. Phys. Chem. Solids, 45, 6. 
643 (1984).

24. A. M. Oles, B. Oles, and K. A. Chao, J. Phys. C13, L979 (1980); J. Rossler and B. Fernández, Phys. Rev. B24, 5299 (1981).

25. L. M. Falicov in Kecent Progress in Many-body Theories Vol. 1, ed. by A. J. Kallio, E. Pajanne, and R. F. Bishop (Plenum Publishing Corp., New York (1988), p. 275 ; J. Callaway, Physica B149, 17 (1988).

26. It could also be thought of as a four-atom 'square' in two dimensions with 'box' boundary conditions, or a square lattice with periodic boundary conditions if the substitution $t \rightarrow 2 t$ is made. It could also be shown to be isomorphic to the four-atom $b c c$ cluster 23 in three dimensions under the substitution $t \rightarrow 4 t$.

27. Bipartite lattices are the ones which break up into two sublattices $A$ and $B$ with non-zero hopping from $A \leftrightarrow B$ but not from $A \leftrightarrow A$ or $B \leftrightarrow B$, so that the energy spectrum is symmetric in $t \leftrightarrow-t, e . g$., the linear chain, square lattice, simple cubic, bcc etc. It is important to note that $f c c$ is not a bipartite lattice.

28. This is one example of the lack of participation of a given- $N$ ground state mentioned in section-I. In this particular case of the tetrahedral, the ground state energies are:

(a) for $x=4.0$ :

$E_{G}(N=2)=-5.29150, E_{G}(N=3)=-3.64681, E_{G}(N=4)=-2.10275$ 
(b) for $x=8.0: E_{G}(N=2)=-4.92820, E_{G}(N=3)=-3.06808, E_{G}$

$$
(N=4)=-1.32023 \text {; }
$$

in both cases (and it is true for all $x>0$ )

$E_{G}(N=3)>\frac{1}{2}\left\{E_{G}(N=2)+E_{G}(N=4)\right\}$.

and the $N=3$ ground state does not contribute to the ensemble averages as $\tau \rightarrow 0$.

29. Any four-atom cluster with a symmetric 1-2-1 one-electron energy structure should exhibit the same behavior; in addition to the linear chain, the isolated square and the simple square (2D) lattice, this property is found in the $b c c$ structure with a sampling of four atoms. For further details see ref. 23 .

30. J. E. Hirsch, Phys. Rev. B31, 4403 (1985).

31. J. E. Hirsch, Phys. Rev. B35, 1851 (1987).

32. The result is first due to E. P. Wigner; for details see ref. 8 .

33. Because of the result regarding the $N=2$ being a very special case where the ground state is spin-unpolarized under very general conditions ${ }^{32}$, the analysis was also done by studying 'flow' in the $N=3$ and $N=4$ subspaces to $n=0.25$. No spin-polarization was observed. 
34. Y. Nagaoka, Solid State Com. 3, 409 (1965); D. J. Thouless, Proc. Phys. Soc. London 86, 893 (1965); Y. Nagaoka, Phys. Rev. 147. 392 (1966); H. Tasaki, Phys. Rev. B40 , 9192 (1989). 


\section{G. Figures for chapter-IV}

Figure 4.1

The "solid" (black) lines where a single $N$ contributes to the ground state, for the four-atom ring and repulsive interactions $(x>0)$. The white areas are regions where more than one value of $N$ contribute. The dashed line indicates the value $x=4.6$ above which the solid lines at $N=3$ and $N=5$ appear. Note the $y$-axis $(\Delta)$ is in the LOG scale. 


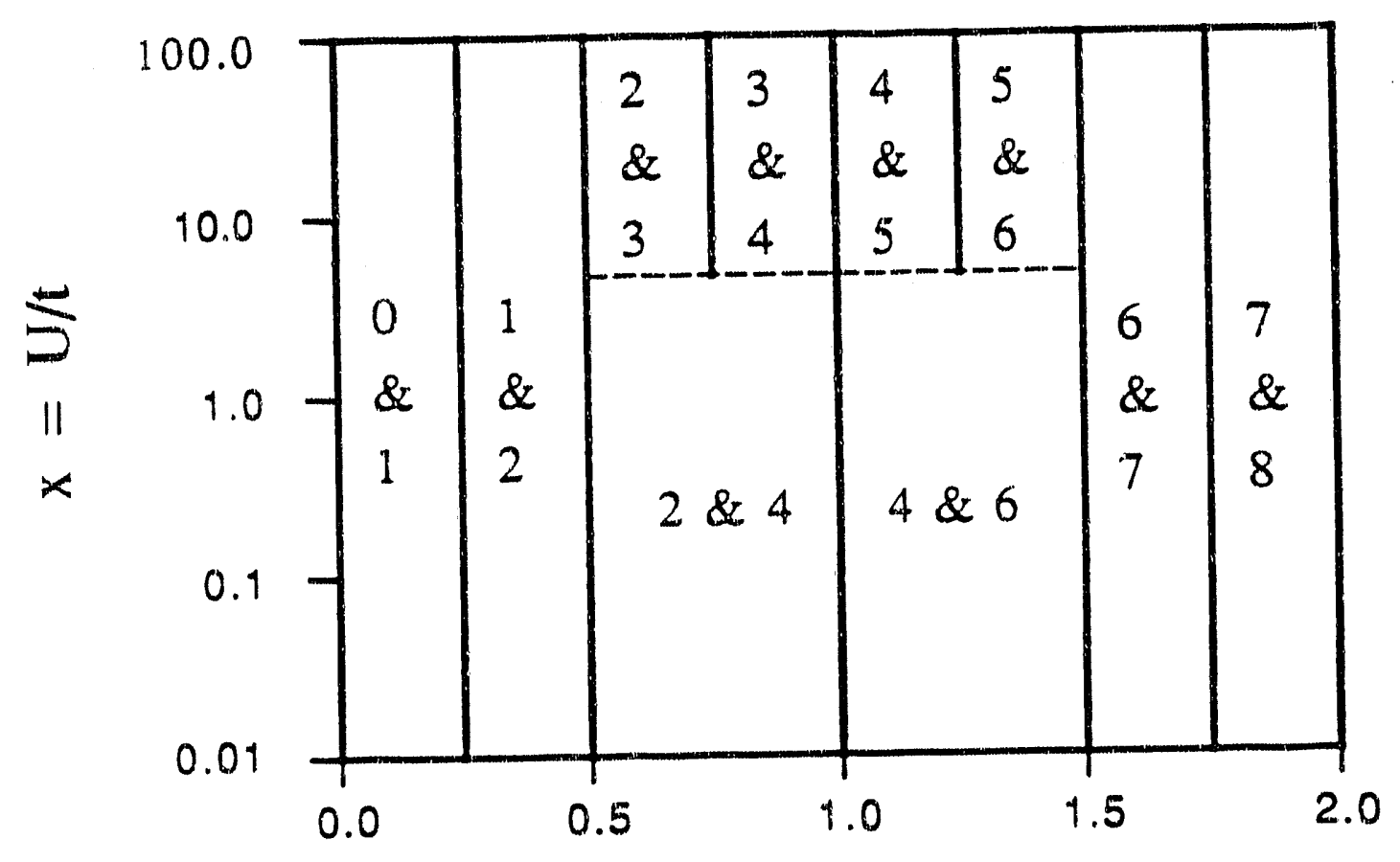

$n=$ fractional occupation 
Figure 4.2

Same as Figure 4.1 for the tetrahedral cluster.

Note the assymetry about half-filling. 


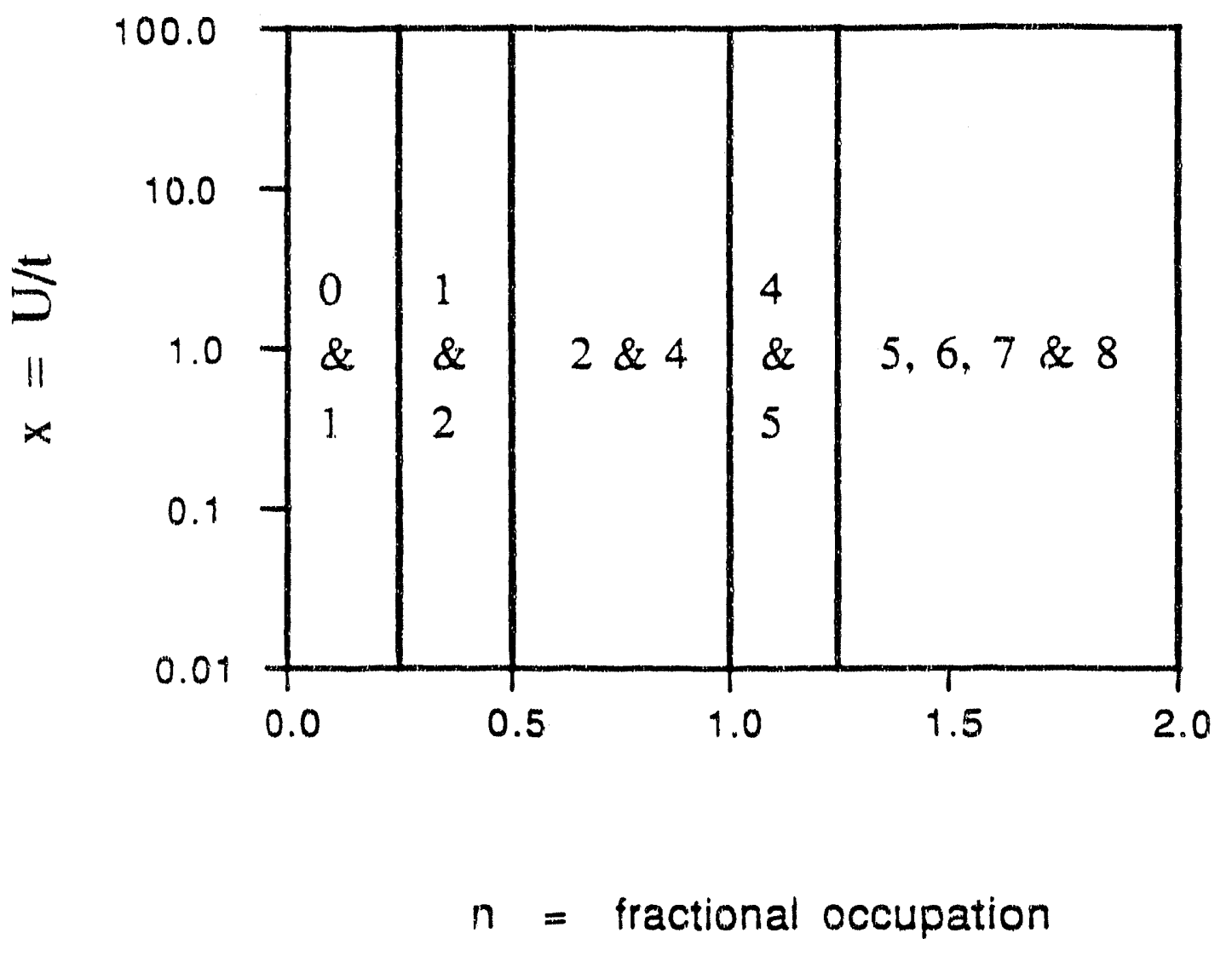


Figure 4.3

Same as Figure 4.1 for either cluster and attractive interactions $(x<0)$. Note only the even number of electrons conform to energetically stable configurations of the system. 


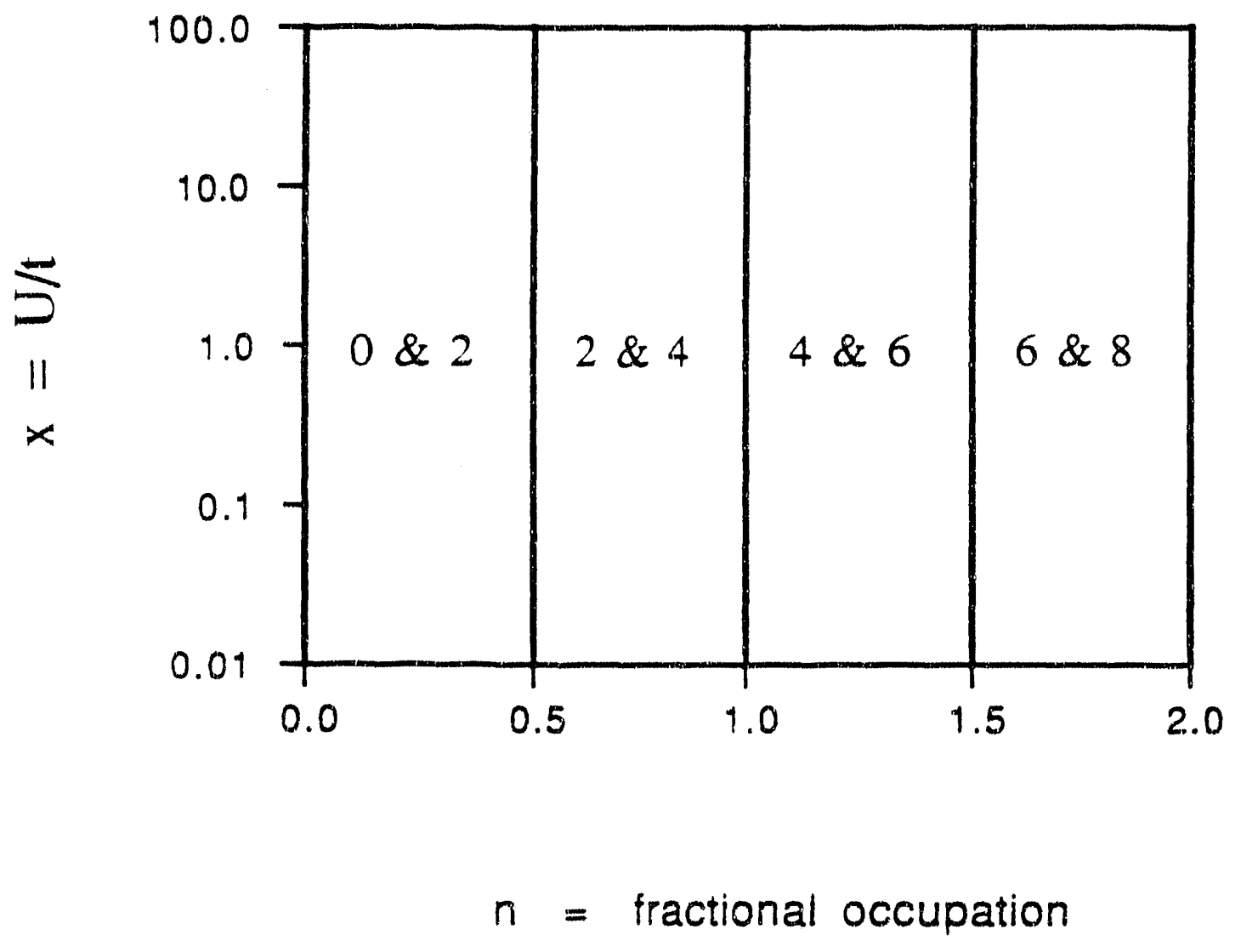


Figure 4.4

Quantum mechanical particle fluctuation $\Delta$ in the four-atom ring at the $\alpha$-site in the exact grand canonical ensemble $(\mu=0)$. "Solid" black lines where discontinuities in $\partial \Delta / \partial n$ occur are drawn. Both attractive and repulsive interactions are considered and values of $x$ are indicated. 


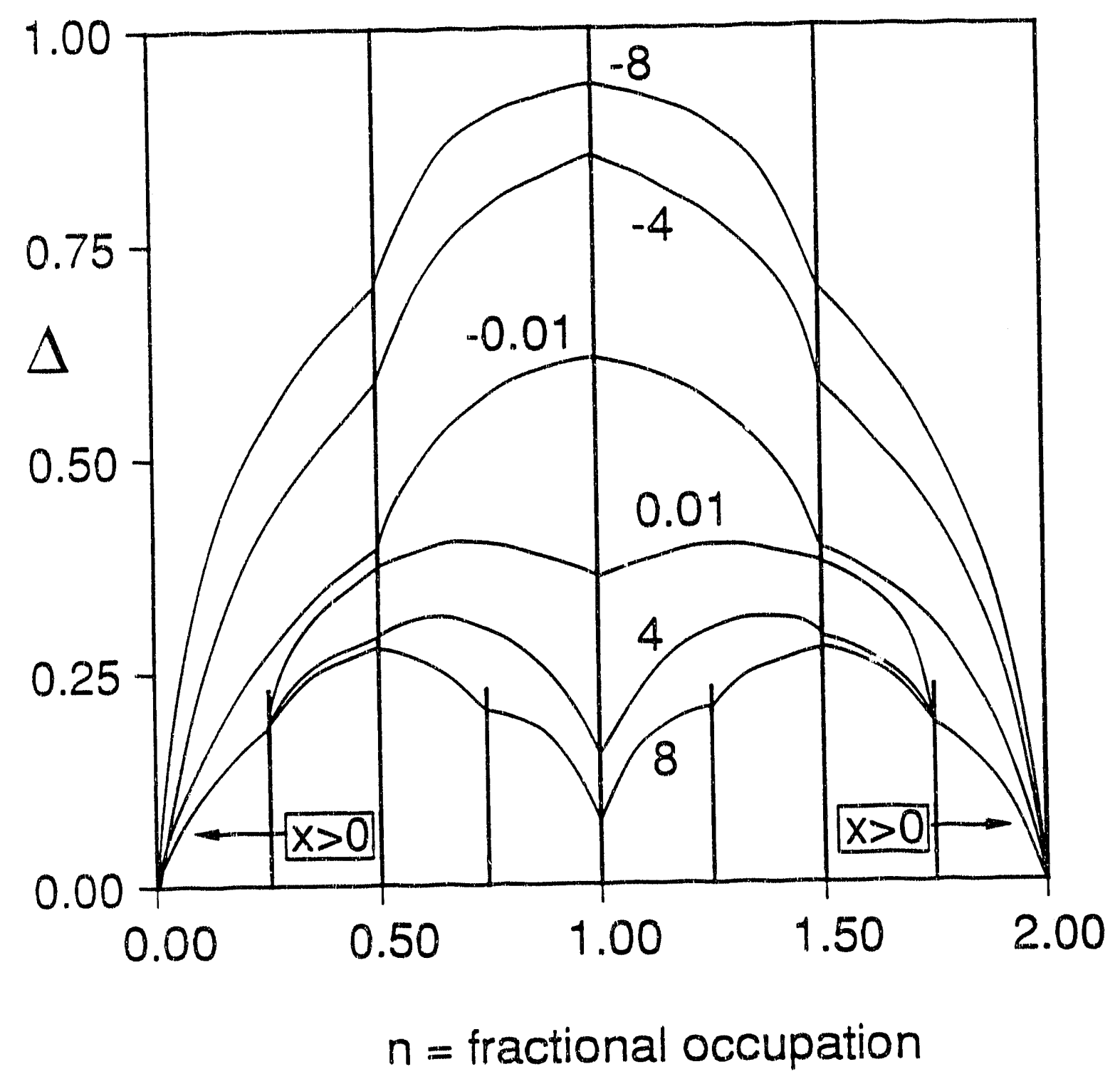


Figure 4.5

Same as Figure 4.4 for the tetrahedral cluster. 


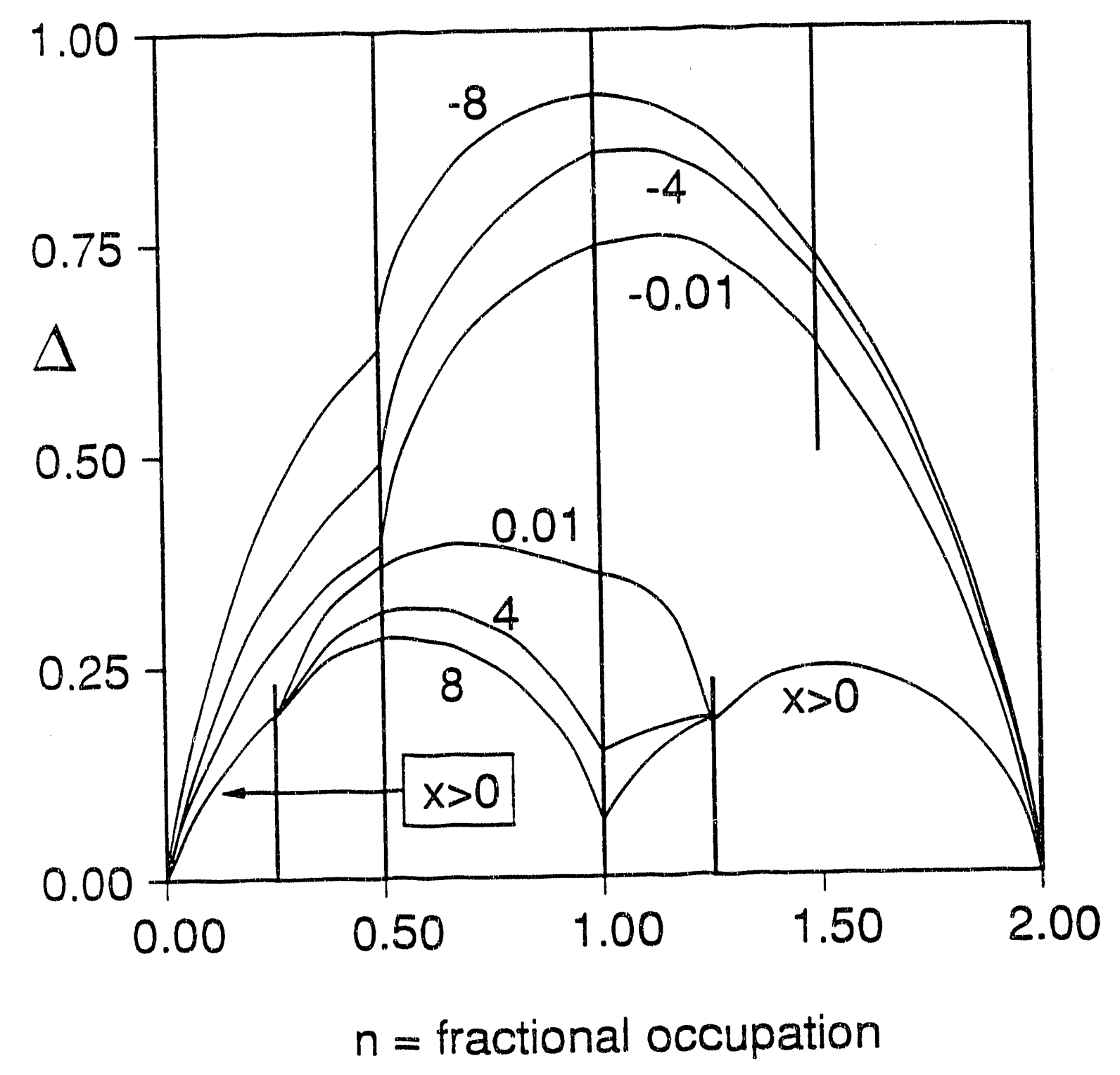



by the variation of the local chemical potential $\mu$ at the $\alpha$-site in the four-atom ring on and around the "solid" black lines of Figure 4. Grey areas are regions away from the solid black lines where the analysis with non-zero $\mu$ is not appropriate. 


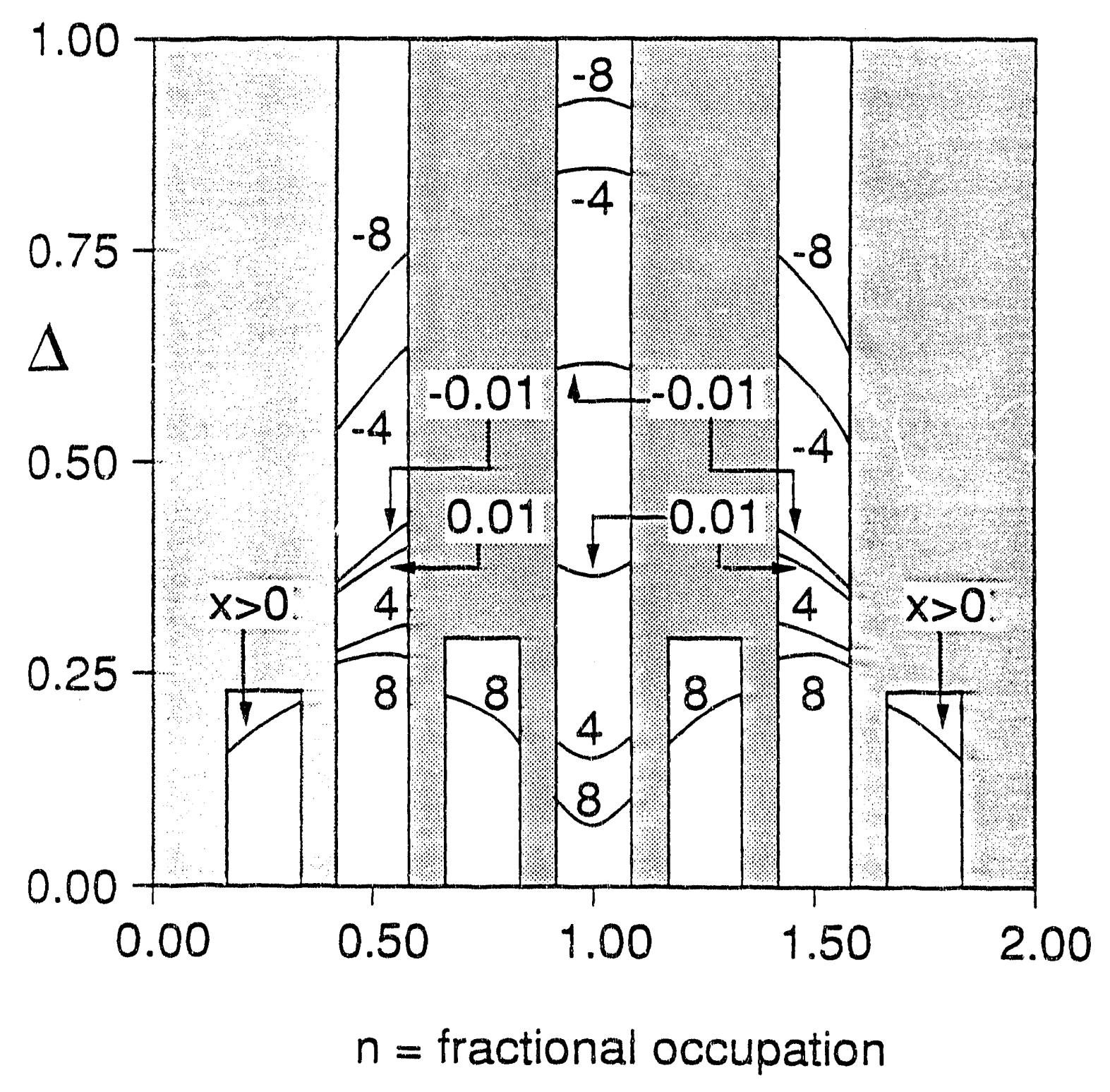


Figure 4.7

Same as Figure 4.6 for the tetrahedral cluster (Figure 4.5). 


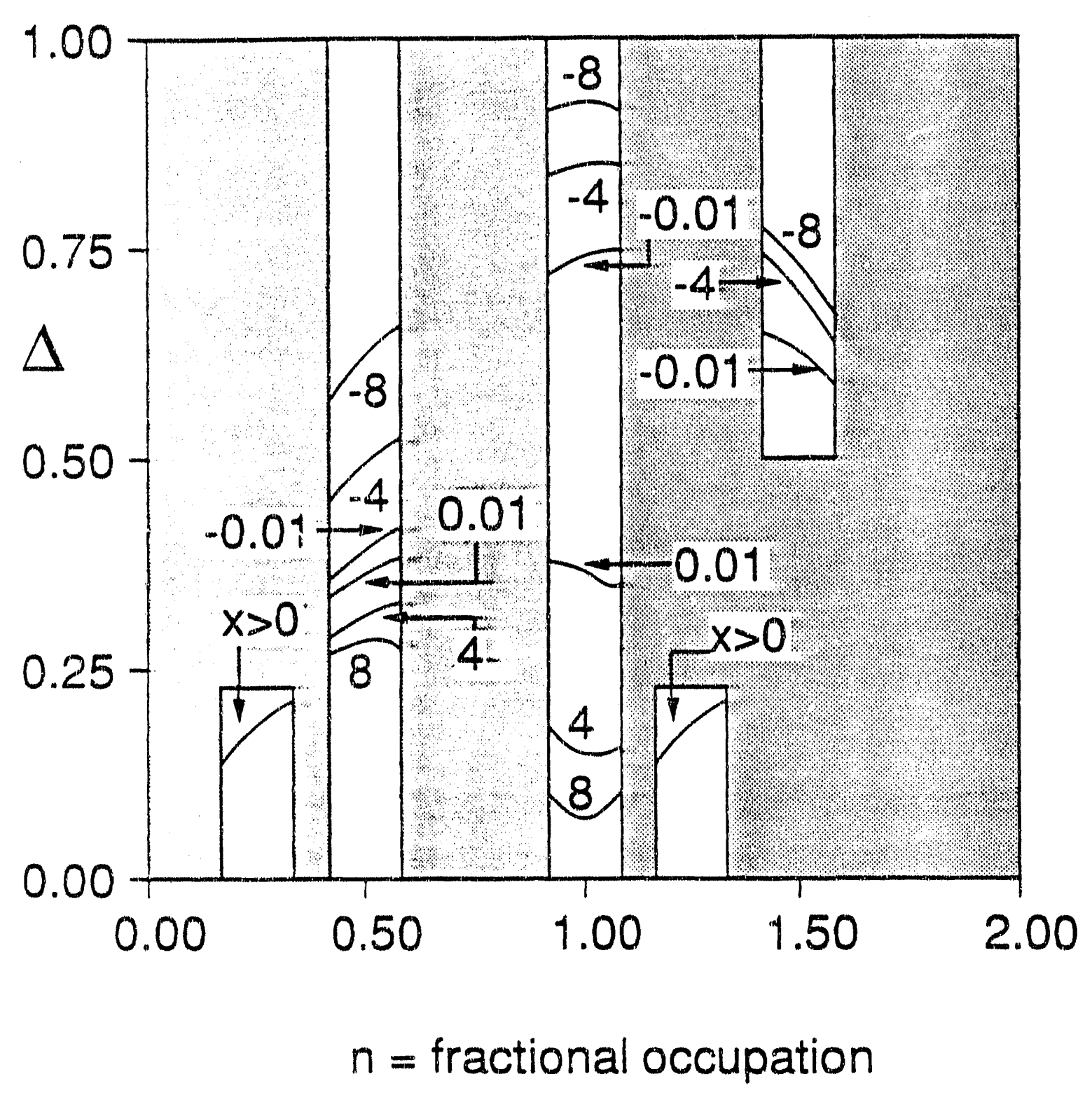



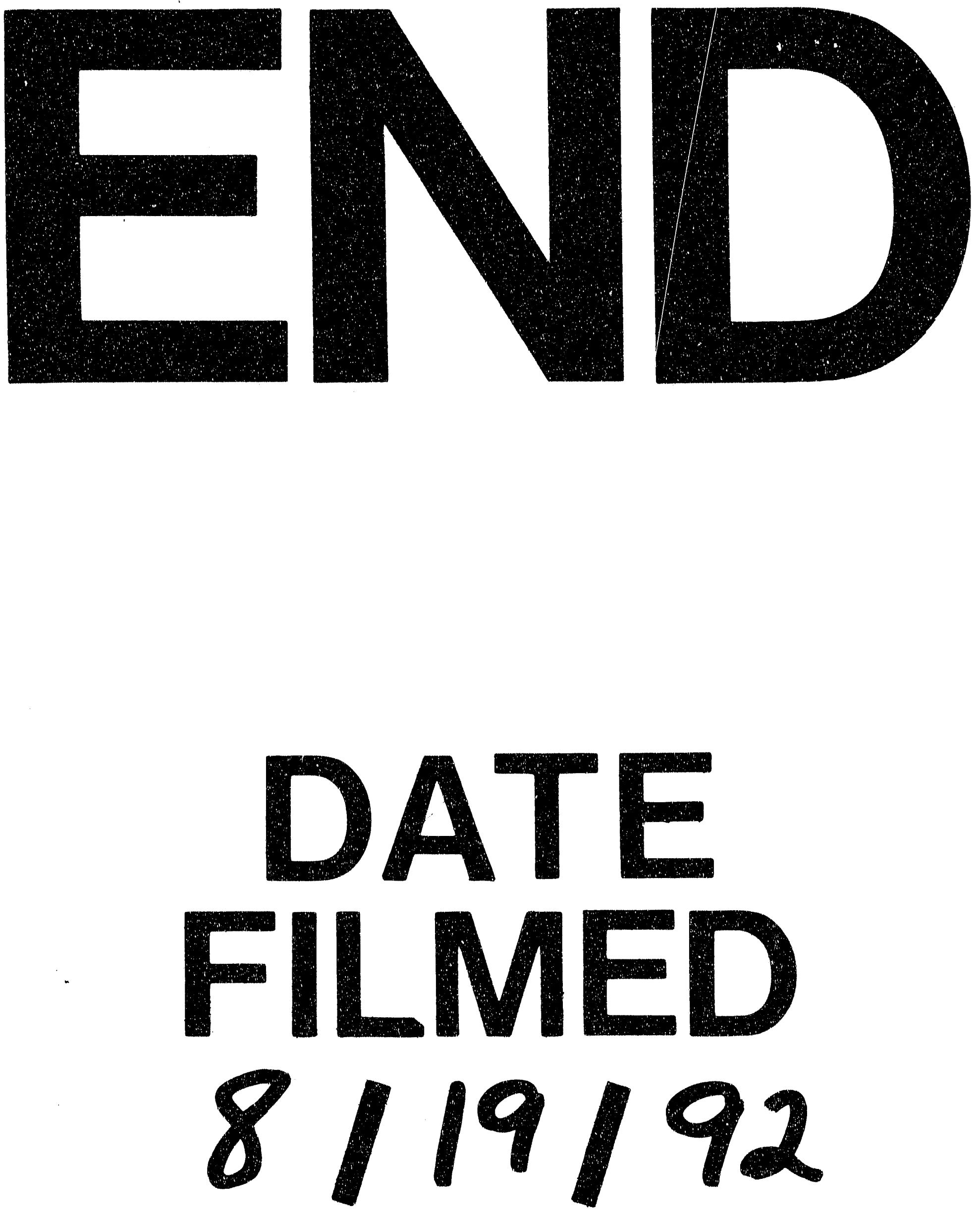
